\title{
Fast Algorithm for Robust Template Matching With M-Estimators
}

\author{
Jiun-Hung Chen, Chu-Song Chen, and Yong-Sheng Chen
}

\begin{abstract}
In this paper, we propose a fast algorithm for speeding up the process of template matching that uses M-estimators for dealing with outliers. We propose a particular image hierarchy called the $p$-pyramid that can be exploited to generate a list of ascending lower bounds of the minimal matching errors when a nondecreasing robust error measure is adopted. Then, the set of lower bounds can be used to prune the search of the $p$-pyramid, and a fast algorithm is thereby developed in this paper. This fast algorithm ensures finding the global minimum of the robust template matching problem in which a nondecreasing M-estimator serves as an error measure. Experimental results demonstrate the effectiveness of our method.
\end{abstract}

Index Terms-Fast algorithm, M-estimator, robust template matching, template matching.

\section{INTRODUCTION}

$\mathbf{F}$ INDING a pattern or template in a signal is an important problem for signal and image processing. This so-called template matching can be applied to many applications such as image and video coding, pattern recognition, and visual tracking. It is usually assumed in template matching that the signal segments of interests do not change their appearances very much. Hence, template matching based on the criteria such as the sum of absolute difference (SAD) or the sum of squared difference (SSD) is commonly adopted. The popularity of using template matching for applications of signal or image processing is mainly due to its ease of implementation together with the many fast algorithms that can be used to speed up the matching process for various applications [1], [7], [8], [13], [15], [22], [25], [26], [28], [29], [35], [39].

In a cluttered environment, however, some outliers such as impulse noises or partial occlusions may occur during the matching processes. In this situation, the SAD and SSD criteria are no longer suitable for template matching because they treat the outliers and inliers evenly when calculating the error measures. One possible remedy for this weakness is to use a robust criterion instead of SAD or SSD. For this, the M-estimator technique [2], [16], [30], [38] is one of the most popular methods to solve the problem of robust parameter estimation and has been applied in many studies [3]-[5],

Manuscript received October 17, 2001; revised August 14, 2002. This work was supported in part by the National Science Council of Taiwan, R.O.C., under Grants NSC 90-2213-E-001-033 and NSC91-2213-E-001-022. The associate editor coordinating the review of this paper and approving it for publication was Dr. Xiang-Gen Xia.

J.-H. Chen and C.-S. Chen are with the Institute of Information Science, Academia Sinica, Taipei, Taiwan, R.O.C. (e-mail: song@iis.sinica.edu.tw).

Y.-S. Chen is with the Integrated Brain Research Laboratory, Department Medical Research and Education, Taipei Veterans General Hospital, Taipei, Taiwan, R.O.C.

Digital Object Identifier 10.1109/TSP.2002.806551
[11], [12], [14], [23], [31]-[33], [36]. The basic idea of the M-estimator technique is to limit the influence of outliers in the matching error. In principle, the effects of the outlier can be suppressed with the M-estimator technique and therefore better estimations are obtained.

A typical procedure for finding solutions with M-estimators is the iterative-reweight procedure [30]. In each iteration of this procedure, a weighted least-square problem is solved and then the weights are adjusted for the next iteration for further refinement. Hence, when applying the iterative-reweight procedure for robust template matching, in each iteration, another template matching problem must be solved based on a weighted SSD error measure, in addition to which, multiple iterations are also necessary. Therefore, the computation of robust error measures is very time-consuming, although more accurate results can be obtained by adopting a robust error measure instead of nonrobust ones. ${ }^{1}$ In the past, many methods have been proposed to speed up the matching process where the simple SAD or SSD criterion is used. However, to our knowledge, no method has been addressed for speeding up the process of template matching where robust error measures are used. In this paper, we propose a fast method for solving this problem. We will present this method by assuming that a two-dimensional (2-D) signal (e.g., an image) is used. Nevertheless, our algorithm can be easily generalized for any $d$-dimensional signal $d \in N^{+}$.

On the other hand, there are already many methods for speeding up the process of template matching where nonrobust error measures are used. These methods can be divided into two classes. The methods in the first class only find a local minimum while the ones in the second class definitely find the global minimum. In principle, almost all the methods in the first class formulate the template matching as a search problem and find a solution by adopting the greedy strategy. Examples include the three-step search algorithm [22], the gradient-descent based method [29] and others [1], [8], [13], [15], [28], [35], [39]. The genetic algorithm-based methods [8], [28] or the simulated annealing-based method [35] may have chances of finding the global minimum if their parameters are set appropriately to the given problems, but can not ensure that it will always be found. In essence, since these methods do not guarantee finding the global minimum, they are generally faster than those ensuring the global optimality.

The methods in the second class guarantee finding the global minimum, and the main idea of this class is basically prune and search [7], [25], [26]. Hence, the main issue of this class of approaches is on how to design the search strategies for pruning

\footnotetext{
${ }^{1}$ Another problem of the iterative-reweight procedure is that it can not definitely find the global optimum.
} 
unnecessary searching branches. The successive elimination algorithm proposed by Li and Salari [26] eliminates impossible sites successively during the searching process by using lower bounds derived from the triangle inequality. Their method can guarantee obtaining the global minimum, as does the full search (FS) method, and it is more efficient. In [25], Lee and Chen extended this idea by using a block-sum-pyramid structure, where a set of ascending lower bounds can be derived and serve as useful guidelines to prune the search process. However, the performance of their method depends on the search order. Recently, Chen et al. further refined it by using a winner-update strategy [7], which is not only irrelevant to the search order but is also faster.

Similar to those methods that ensure finding the global minimum but using nonrobust error measures introduced above [7], [25], [26], the method developed in this paper also adopts an inequality in a particular image hierarchy to speed up the templatematching process with robust error measures. In essence, a set of ascending lower bounds of the minimal matching error can be generated with our method as long as the robust error measure is nondecreasing. This set of lower bounds can then serve as useful guidelines for pruning the redundant branches of the searching process. In addition, our method can ensure finding the global minimum, as the FS method does for robust template matching.

This paper is organized as follows. Section II introduces the image hierarchy used in this work and the associated ascending lower bound list. Section III presents the search strategies and our main algorithm. Section IV shows some experimental results. Finally, some discussion and conclusions are given in Sections V and VI, respectively.

\section{Problem Formulation, $P$-Pyramid, AND FUNDAMENTAL INEQUALITY}

\section{A. Problem Formulation}

We denote $I(i, j)$ as the intensity at position $(i, j)$ in an image $I$. Assume that $I_{1}$ and $I_{2}$ are $N \times N$ images. The sum of robust differences (SRD) between two images $I_{1}$ and $I_{2}$ is defined as follows:

$$
\operatorname{SRD}_{\rho, \sigma}\left(I_{1}, I_{2}\right) \equiv \sum_{0 \leq i, j \leq N-1} \rho\left(\left|I_{1}(i, j)-I_{2}(i, j)\right|, \sigma\right)
$$

where $\rho(\cdot, \cdot): R^{+} \cup\{0\} \times R^{+} \rightarrow R^{+} \cup\{0\}$ is a robust error measure (or a robust loss function) [2], [16], [38], and $\sigma$ is a parameter controlling the shape of $\rho(\cdot, \cdot) .^{2}$ Typically, the robust error measure, $\rho(\cdot, \cdot)$, is selected according to how it reduces the influences of outliers. Given an image template $I_{t}$ whose size is $N \times N$ and an image $F$ whose size is $(2 W+N) \times(2 W+N)$, the robust template matching problem is defined as finding the position $\left(u^{*}, v^{*}\right)$ with the minimum SRD among all possible search positions in the image $F$

$$
\begin{aligned}
\left(u^{*}, v^{*}\right) \equiv & \arg \min _{-W \leq u, v \leq W} \operatorname{SRD}_{\rho, \sigma}\left(I_{t}, F_{u, v}\right) \\
= & \arg \min _{-W \leq u, v \leq W} \sum_{0 \leq i, j \leq N-1} \\
& \cdot \rho\left(\left|I_{t}(i, j)-F(i+u, j+v)\right|, \sigma\right)
\end{aligned}
$$

${ }^{2}$ Some common robust error measures will be introduced in Section II-E. In particular, if $\rho(x, \sigma)=x^{2}$ (or $\left.x\right)$ for all $\sigma$, then (1) becomes SSD (or SAD). where $F_{u, v}$ is an $N \times N$ image block with its upper-left point being $(u, v)$ in the image $F$, and the number of search sites is $(2 W+1) \times(2 W+1)$.

\section{B. P-Feasible}

To simplify the notation of the robust error measures, we define

$$
\tau_{\sigma}(x)=\rho(x, \sigma) \quad \text { for all } x \in R^{+} \cup\{0\}
$$

and abbreviate $\tau_{\sigma}(x)$ to be $\tau(x)$ for the cases without ambiguity in the following.

Definition 1 [p-Feasible]: A robust error measure $\tau(\cdot)$ is $p$-feasible if it satisfies both the following two conditions.

1) $\tau(\cdot)$ is nondecreasing, i.e., $a_{1} \geq a_{2}$ implies $\tau\left(a_{1}\right) \geq$ $\tau\left(a_{2}\right)$ for all $a_{1}, a_{2} \in R^{+} \cup\{0\}$.

2) For each pair of nonnegative values $\left(a_{1}, a_{2}\right)$, the following inequality with respect to the $L_{p}$-norm holds:

$\forall a_{1}, a_{2} \in R^{+} \cup\{0\}, \quad \tau\left(a_{1}\right)+\tau\left(a_{2}\right) \geq \tau\left(\|\mathbf{a}\|_{p}\right)$ where $\mathbf{a}=\left[\begin{array}{ll}a_{1} & a_{2}\end{array}\right]^{T}$ is a 2-D vector, and $\|\mathbf{a}\|_{p}=\left(\left|a_{1}\right|^{p}+\right.$ $\left.\left|a_{2}\right|^{p}\right)^{1 / p}$ is the $L_{p}$-norm of a, $p \in[1 \infty]$. In particular, $\|\mathbf{a}\|_{\infty}=\max \left(\left|a_{1}\right|,\left|a_{2}\right|\right)$.

Some properties associated with the $p$-feasible defined above are investigated in the following.

Property 1: Every nondecreasing $\tau(\cdot)$ is $\infty$-feasible.

Proof: This property can be easily derived according to the definitions.

Property 2: If a robust error measure $\tau(\cdot)$ is $p$-feasible, that implies it is also $q$-feasible for all $q \in[p \infty]$.

Proof: See Appendix A.

If a $p$-feasible robust error measure is used as the matching criterion, an ascending lower bound list of the matching errors can be obtained by constructing an image pyramid with respect to the $L_{p}$-norm, as described in the following.

\section{P-Pyramid and Fundamental Inequality}

Assume that $N=2^{n}\left(n \in N^{+}\right)$. For each $N \times N$ image block $I=F_{u, v}(-W \leq u, v \leq W)$ that is contained in the image $F$, a $p$-pyramid of $I$ is defined as a set of images $\left\{I^{0, p}, \ldots, I^{m-1, p}, I^{m, p}, \ldots, I^{n, p}\right\}$, where $I^{n, p}=I$ and the size of $I^{m, p}$ is $2^{m} \times 2^{m}\left(0 \leq m \leq n, m \in N^{+} \cup\{0\}\right)$. $I^{m, p}$ is referred to as the image on the level $m$ of the $p$-pyramid. Level 0 and level $n$ are called the highest and the lowest levels of the $p$-pyramid, respectively. Given an image $I^{m, p}$ on the level $m$, the image $I^{m-1, p}$ on the level $m-1$ is constructed using the following equation:

$$
I^{m-1, p}(i, j)=\left\|\mathbf{I}_{i, j}^{m, p}\right\|_{p}
$$

where

$$
\begin{array}{r}
\mathbf{I}_{i, j}^{m, p}=\left[\begin{array}{ll}
I^{m, p}(2 i, 2 j) & I^{m, p}(2 i, 2 j+1) \quad I^{m, p}(2 i+1,2 j) \\
I^{m, p}(2 i+1,2 j+1)
\end{array}\right]^{T} \text { and } 0 \leq i, j \leq 2^{m-1}-1 .
\end{array}
$$

Accordingly, the pyramids from level $n$ to level 0 can be constructed iteratively. Totally, $(2 W+1) \times(2 W+1) p$-pyramids are constructed from the image $F$. Fig. 1 shows an illustration of the pyramids constructed from a one-dimensional (1-D) signal.

Following the notions shown in (1), we define the SRD between $I_{1}$ and $I_{2}$ on the level $m$ by $\operatorname{SRD}_{\rho, \sigma}^{m, p}\left(I_{1}, I_{2}\right)=$ 
$\operatorname{SRD}_{\rho, \sigma}\left(I_{1}^{m, p}, I_{2}^{m, p}\right)$ for $m=0,1, \ldots, n$. Furthermore, we denote $\operatorname{SRD}_{\tau}\left(I_{1}, I_{2}\right)=\operatorname{SRD}_{\rho, \sigma}\left(I_{1}, I_{2}\right)$ if a fixed $\sigma$ is considered [where $\tau(\cdot)$ is defined in (3)]. Then, it can be shown that the following fundamental inequality holds for the image hierarchy defined above.

Theorem 1: Given a robust error measure $\tau(\cdot)$ that is $p$-feasible, then for all $I_{1}, I_{2}$,

$$
\begin{aligned}
& \operatorname{SRD}_{\tau}\left(I_{1}^{n, p}, I_{2}^{n, p}\right) \\
& \quad \geq \operatorname{SRD}_{\tau}\left(I_{1}^{n-1, p}, I_{2}^{n-1, p}\right) \geq \cdots \geq \operatorname{SRD}_{\tau}\left(I_{1}^{0, p}, I_{2}^{0, p}\right) .
\end{aligned}
$$

\section{Proof: See Appendix B.}

Hence, given a image template $I_{t}$ and an image block $F_{u, v}(-W \leq u, v \leq W)$, a set of ascending lower bounds, $\operatorname{SRD}_{\tau}^{m, p}\left(F_{u, v}, I_{t}\right), m=0,1, \ldots, n$ can be obtained according to Theorem 1.

Notice that the number of robust differences involved in the computation of each lower bound $\operatorname{SRD}_{\tau}^{m, p}\left(F_{u, v}, I_{t}\right)$ $(0 \leq m<n)$ is $2^{m} \times 2^{m}$, which is smaller than $2^{n} \times 2^{n}$, the number of robust differences required forSRD ${ }_{\tau}^{n, p}\left(F_{u, v}, I_{t}\right)$. Therefore, the lower bounds can be computed more efficiently than the sum of robust differences with respect to the original image. In particular, the higher are the levels in a pyramid, the faster are the computations of the associated lower bounds. In fact, the ratio of the required number of the computations of the robust differences on the level $m$ to that required for the level $n$ is $1 / 4^{n-m}$. Accordingly, even when all of the lower bounds are computed, the required time is less than $\sum_{m=1}^{n-1}\left(1 / 4^{n-m}\right) \leq 1 / 3$ of that required for the level $n$, the original image block. Such an ascending lower bound list of the matching error can be used for speeding up the matching process by incorporating it into a systematic search strategy, as introduced in Section III.

Remark 1 [Efficient Construction of the P-Pyramids of an Image]: A $p$-pyramid of each image block can be constructed independently by using (5). However, it is very time-consuming if each pyramid is constructed independently. In fact, the $p$-pyramids can be constructed more efficiently by considering the computation and storage redundancies between neighboring image blocks when constructing and storing the $p$-pyramids. We introduce this method by using an example, as shown in Fig. 1. Considering node $A$ in the first level image of Pyramid 1, as shown in Fig. 1, one can observe that node $A$ is also contained in Pyramid 3. Similarly, node B (or C) is shared by Pyramids 2 and 4 (or Pyramids 3 and 5). Hence, if each pyramid is constructed independently according to (5), the values of nodes $\mathrm{A}, \mathrm{B}$, and $\mathrm{C}$ will be computed twice: once for each pyramid. In our work, to remove this redundancy and to save computation and storage of the $p$-pyramids for all the image blocks, the method illustrated in Fig. 1 is adopted. That is, $I^{1}$, the image containing every node of the first-level images of all the pyramids, is first constructed based on $I^{2}$. Similarly, $I^{0}$ can be constructed based on $I^{1}$. After $I^{0}$ and $I^{1}$ are constructed, all the pyramids are then available, as shown in Fig. 1. More details about efficient construction of pyramids can be found in [7].

Remark 2 [Free Sampling]: In the above description, a particular level of the $p$-pyramid is built with the $L_{p}$-norm of the $2 \times 2$

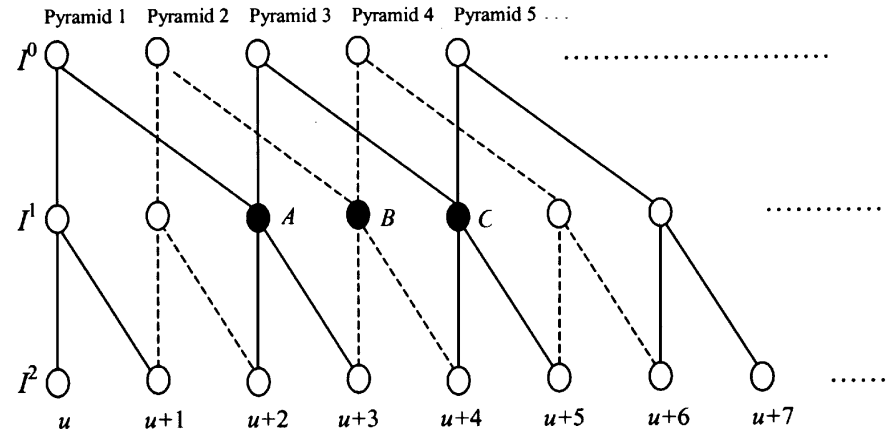

Fig. 1. $\quad p$-pyramid constructed from a 1-D signal where each element in higher levels is composed of its two son elements. Five $p$-pyramids are depicted in solid or dashed lines. The three black nodes are the ones that are shared between two pyramids.

points from its lower neighbor level, as shown in (5). We refer to it as the 2-2 downsampling in this case. In general, we can use $m-n$ downsampling instead of 2-2 downsampling for all $m, n \in N^{+}$, and the associated $p$-pyramid can be reconstructed in a similar way as well. Similarly, $m$ downsampling can be used for a 1-D signal and $m-n-k$ downsampling can be used for a three-dimensional (3-D) signal, and so on, where $k \in N^{+}$.

\section{Characterization of P-Feasible Robust Error Measures}

In Section II-C, we have shown that if a $p$-feasible robust error measure is selected for template matching, then we can construct an ascending lower bound list associated with a particular image hierarchy: the $p$-pyramid. Before introducing the search procedure that exploits the series of lower bounds in detail, we illustrate an important issue about whether such a lower-bound list can be constructed. In particular, the following problem is worthy of being addressed.

"Given a robust error measure $\tau(\cdot)$, under what condition can it be ensured that we can always find a $p \in[1 \infty]$ such that $\tau(\cdot)$ is $p$-feasible?"

In this paper, we tackle this problem in consideration of the class of nondecreasing robust error measures. In fact, Property 1 has shown that if a robust error measure is nondecreasing, it is $\infty$-feasible. Therefore, when the $\infty$-pyramids are built for both the template and the image blocks, an ascending lower bound list can then be constructed according to Theorem 1. Hence, it ensures that every nondecreasing robust error measure is $p$-feasible for some $p$ because $p=\infty$ is a trivial solution. ${ }^{3}$ In addition to $p=\infty$, let us further investigate the other $p$ values that allow a robust error measure to be $p$-feasible. Given a nondecreasing robust error measure, $\tau(\cdot)$, let $\Gamma_{\tau}$, which is the feasible set associated with $\tau$, be defined as the set of values allowing $\tau(\cdot)$ to be $p$-feasible: $\Gamma_{\tau}=\{p \in[1 \infty] \mid \tau$ is $p$-feasible $\}$. By considering the maximal lower bound of $\Gamma_{\tau}$, the following property can be derived.

Property 3: Given a $\tau(\cdot)$ that is nondecreasing, there exists a discriminative value $p^{\prime}$ such that $\tau(\cdot)$ is $p$-feasible for all $p \in$ $\left(\begin{array}{ll}p^{\prime} & \infty\end{array}\right]$ and is not $p$-feasible for all $p \in\left[1 p^{\prime}\right)$, where $p^{\prime}$ is the maximal lower bound of $\Gamma_{\tau}$, which is the feasible set associated with $\tau$.

${ }^{3}$ How to construct a list of ascending lower bounds for the general class of robust error measures that are not necessary to be nondecreasing remains an open problem. 
Proof: This property can be derived directly from Properties 1 and 2.

Hence, the feasible set associated with a robust error measure can be clearly specified with its discriminative value $p^{\prime}$ by further identifying the following two conditions: $\tau$ is $p^{\prime}$-feasible or $\tau$ is not $p^{\prime}$-feasible. If $\tau$ is $p^{\prime}$-feasible, then $\Gamma_{\tau}=\left[p^{\prime} \infty\right]$; otherwise, $\Gamma_{\tau}=\left(p^{\prime} \infty\right]$. After specifying the feasible set, another problem worth consideration is the following.

"Which $p$ contained in the feasible set associated with a nondecreasing robust error measure is a better choice for speeding up the process of robust template matching?"

We investigate the above problem from the implementation point of view. In practice, to simplify the computation, it is better to select $p$ as integersinstead of floating-point numbers. When $p$ is restricted to being an integer, the feasible set associated with $\tau(\cdot)$ can then be uniquely specified by $\Gamma_{\tau}=\left\{n \in N^{+} \cup\{\infty\} \mid n \geq n^{\prime}\right.$, $\left.n^{\prime} \in N^{+} \cup\{\infty\}\right\}$, where $n^{\prime}=\left\lceil p^{\prime}\right\rceil$. In particular, we call $\tau(\cdot)$ to be minimal $n^{\prime}$-feasible in this case, and $n^{\prime}$ is also referred to as the minimal feasible value of $\tau(\cdot)$. Note that the smaller is $n$, the less is the computational overhead of the $L_{n}$-norm for $n \in N^{+}$. Therefore, a better choice of $n$ is therefore $n=n^{\prime}{ }^{4}$

\section{E. Minimal Feasible Value of Commonly Used Robust Error Measures}

In the following, we will give a study of the minimal feasible values for some commonly used robust error measures. An interesting phenomenon shown below is that almost all commonly used nondecreasing robust error measures are minimal 1-feasible or minimal 2-feasible.

Given a robust error measure $\rho(\cdot, \cdot)$, we define $H_{\rho}=\left\{\rho(\cdot, \sigma) \mid \sigma \in R^{+}\right\}$. The Huber's estimator $\rho_{1}(\cdot, \cdot)$ [see Fig. 2(a)] has least squares behaviors for small residues, and the more robust least-absolute-values behavior for large residues [14], [19], [31], [33]:

$$
\rho_{1}(r, \sigma)= \begin{cases}\frac{r^{2}}{2}, & \text { if } r \leq \sigma \\ \sigma\left(r-\frac{\sigma}{2}\right), & \text { otherwise. }\end{cases}
$$

Property 4: Each member of Huber's estimators $H_{\rho_{1}}$ is minimal 2-feasible.

Proof: See Appendix C.

The Tukey's estimator $\rho_{2}(\cdot, \cdot)$ [see Fig. 2(b)] has zero weights ${ }^{5}$ for the large residues and thus improves the outlier rejection properties [4], [5], [11], [14], [19], [31]

$$
\rho_{2}(r, \sigma)= \begin{cases}\frac{\sigma^{2}}{6}\left[1-\left(1-\left(\frac{r}{\sigma}\right)^{2}\right)^{3}\right], & \text { if } r \leq \sigma \\ \frac{\sigma^{2}}{6}, & \text { otherwise. }\end{cases}
$$

The shape of the function $\rho_{2}(\cdot, \cdot)$ is shown in Fig. 2(b).

\footnotetext{
${ }^{4}$ Although the computation of the $\infty$-norm is also simple since only the absolute values and the $\max (\cdot, \cdot)$ operations are involved, we find that in practice its speedup performance is usually worse because the lower bounds associated with an $\infty$-pyramid are usually not tight enough. An example is given in Section IV-A

${ }^{5}$ In robust statistics, the weight is defined to be an value proportional to the derivatives of $\rho$.
}
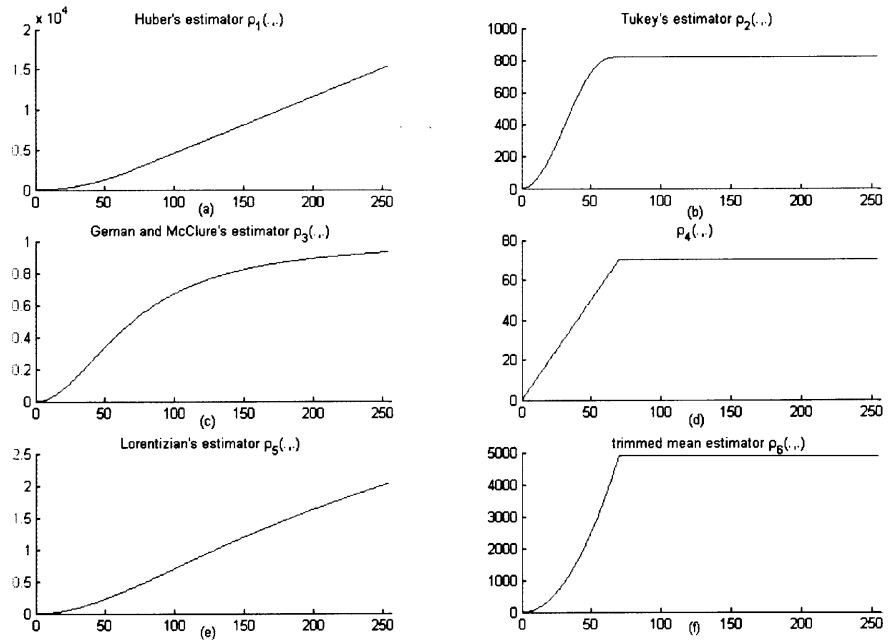

Fig. 2. Shapes of commonly used M-estimators with threshold $\sigma=70$. (a) The shape of $\rho_{1}$. (b) The shape of $\rho_{2}$. (c) The shape of $\rho_{3}$. (d) The shape of $\rho_{4}$. (e) The shape of $\rho_{5}$. (f) The shape of $\rho_{6}$.

Property 5: Each member of Tukey's estimators $H_{\rho_{2}}$ is minimal 2-feasible.

Proof: See [6].

Another popular class of the robust error measure is the one proposed by Geman and McClure [12] [see Fig. 2(c)] as shown below, which was also adopted in [3], [4], [23], and [31]

$$
\rho_{3}(r, \sigma)=\frac{r^{2}}{r^{2}+\sigma^{2}} .
$$

Property 6: Each member of Geman and McClures' estimators $H_{\rho_{3}}$ is minimal 2-feasible.

Proof: See [6].

The above three robust error measures are popular, and we have shown that all members of their $H_{\rho}$ s have the minimal feasible value as 2 . In addition, the following three robust error measures are also investigated.

The robust function $\rho_{4}(\cdot, \cdot)$ [36] [see Fig. 2(d)] uses simple truncations to remove outliers, as shown in the following:

$$
\rho_{4}(r, \sigma)= \begin{cases}r, & \text { if } r \leq \sigma \\ \sigma, & \text { otherwise }\end{cases}
$$

Another two robust error measures investigated here are Lorentizian's estimator $\rho_{5}(\cdot, \cdot)[19]$ [see Fig. 2(e)] and the trimmed mean M-estimator $\rho_{6}(\cdot, \cdot)$ [31] [see Fig. 2(f)]:

$$
\begin{aligned}
& \rho_{5}(r, \sigma)=\log \left(1+\frac{1}{2}\left(\frac{r}{\sigma}\right)^{2}\right) \\
& \rho_{6}(r, \sigma)= \begin{cases}\frac{r^{2}}{2}, & \text { if } r \leq \sigma \\
\frac{\sigma^{2}}{2}, & \text { otherwise. }\end{cases}
\end{aligned}
$$

Property 7: i) Each member of $H_{\rho_{4}}$ is minimal 1-feasible. ii) Each member of $H_{\rho_{5}}$ is minimal 2-feasible. iii) Each member of $H_{\rho_{6}}$ is minimal 2-feasible.

Proof: See [6].

Notice that all the minimal feasible values of the commonly used nondecreasing robust error measures investigated above are 1 or 2 , which are indeed small values. 
In the following, we investigate an M-estimator that is not nondecreasing (the triweight M-estimator $\rho_{7}(\cdot, \cdot)$ ) [32]:

$$
\rho_{7}(r, \sigma)= \begin{cases}r^{2}, & \text { if } r \leq \sigma \\ \sigma^{2}, & \text { if } \sigma<r \leq 3 \sigma \\ 0, & \text { otherwise. }\end{cases}
$$

Since each member of $H_{\rho_{7}}$ is not nondecreasing, the process of template matching can not be speeded up with our approach if $\rho_{7}$ is selected to be the robust error measure.

Finally, we investigate the nondecreasing error measure shown as follows, where $k \in N^{+}$:

$$
\rho_{8}(r, \sigma)=r^{k}
$$

Property 8: Each member of $H_{\rho_{8}}$ is minimal $k$-feasible.

Proof: The proof is trivial.

When $k=1$ (or $k=2$ ), $\rho_{8}$ becomes the SAD (or SSD) error measure. The SAD and SSD are therefore minimal 1-feasible and minimal 2-feasible, respectively. Hence, our method can also be used to speed up the template matching process where $\mathrm{SAD}$ or SSD is used as the error measure.

\section{SEARCh StRATEGy AND THE MAIN AlgORITHM}

\section{A. Search Strategy}

Once an ascending lower bound list of the matching error is available for every search position $(u, v)$, many search strategies [7], [25], [26] can be used to speed up the process of robust template matching in our work. A brief review of these search strategies is given below. Without loss of generality, the $p$-pyramid serves as the pyramidal structure for describing these methods. We refer to p-pyramid as pyramid in the following. Consider a template $I_{t}$ and a set of image blocks $F_{u, v}(-W \leq$ $u, v \leq W)$ to be matched, where $I_{t}$ and $F_{u, v}$ are both $N \times N$ images. Assume that the associated $p$-pyramids have been constructed for $I_{t}$ and all of the $F_{u, v},-W \leq u, v \leq W$, respectively.

In [26], only the highest level and the lowest level of the pyramids were used. The search order of the matching process is fixed and, without loss of generality, assume that the matching process starts from $F_{-W},-W$ and the search is performed in a row-major order. First, the error $\operatorname{SRD}_{\tau}\left(F_{-W,-W}, I_{t}\right)$ is computed as a reference value $r$. Then, assume that $\left(u^{\prime}, v^{\prime}\right)$ is the next site to be visited in the matching order. We try to find out whether the robust error measure $\operatorname{SRD}_{\tau}\left(F_{u^{\prime}, v^{\prime}}, I_{t}\right)$ is smaller than $r$. We do not compute $\operatorname{SRD}_{\tau}\left(F_{u^{\prime}, v^{\prime}}, I_{t}\right)$ directly. Instead, we first compute $\operatorname{SRD}_{\tau}^{0, p}\left(F_{u^{\prime}, v^{\prime}}, I_{t}\right)$, which is the error associated with the highest levels of the pyramids of $I_{t}$ and $F_{u^{\prime}, v^{\prime}}$. If $\operatorname{SRD}_{\tau}^{0, p}\left(F_{u^{\prime}, v^{\prime}}, I_{t}\right)$ is larger than the current reference value $r$, we do not have to further compute $\operatorname{SRD}_{\tau}\left(F_{u^{\prime}, v^{\prime}}, I_{t}\right)$ because $\operatorname{SRD}_{\tau}^{0, p}\left(F_{u^{\prime}, v^{\prime}}, I_{t}\right)$ is a lower bound of $\operatorname{SRD}_{\tau}\left(F_{u^{\prime}, v^{\prime}}, I_{t}\right)$. Therefore, early in the process, we can jump out of the process of matching $I_{t}$ and $F_{u^{\prime}}, v^{\prime}$ and go on to match the next image block in the row-major order. On the other hand, if $\operatorname{SRD}_{\tau}^{0, p}\left(F_{u^{\prime}, v^{\prime}}, I_{t}\right)$ is smaller than the current reference value $r$, we must compute $\operatorname{SRD}_{\tau}\left(F_{u^{\prime}, v^{\prime}}, I_{t}\right)$ and compare it with $r$. If $\operatorname{SRD}_{\tau}\left(F_{u^{\prime}, v^{\prime}}, I_{t}\right)$ is smaller than $r$, the current reference value, then $r$ is replaced by $\operatorname{SRD}_{\tau}\left(F_{u^{\prime}, v^{\prime}}, I_{t}\right)$.

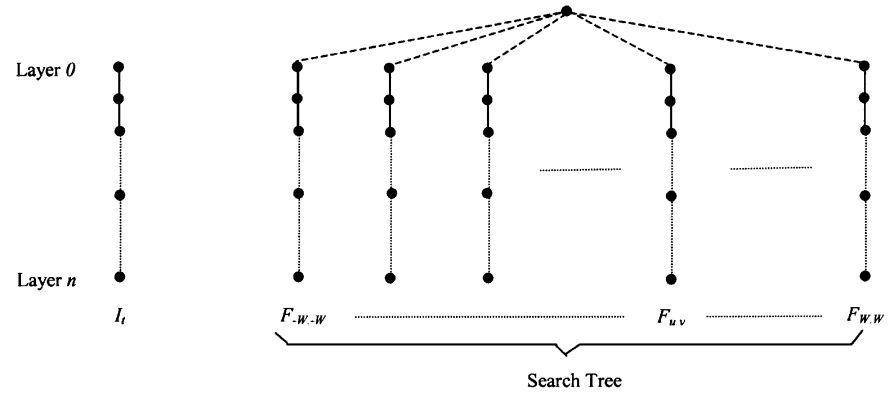

Fig. 3. Search strategies introduced in Section III-A. Li and Salari method [26] only searches the layer 0 and layer $n$ of the tree in a depth-first order. Lee and Chen method [25] searches the entire tree in a depth-first order. Both methods prune the search branches by comparing the current reference value with the error associated with the vertex. Chen et al. method [7] uses the uniform cost search [34] (the branch-and-bound strategy) for the entire tree to prune the unnecessary search branches.

The above procedure can be repeated iteratively. Remember that the computation complexity of $\operatorname{SRD}_{\tau}^{0, p}\left(F_{u^{\prime}, v^{\prime}}, I_{t}\right)$ is $1 / 4^{n}$ of that required for $\operatorname{SRD}_{\tau}\left(F_{u^{\prime}, v^{\prime}}, I_{t}\right)$, and thus, such an early jump-out effect saves considerable computation time.

Lee and Chen [25] extended the idea of [26], using not only the highest and the lowest levels, but all the levels of the pyramids. First, the error $\operatorname{SRD}_{\tau}\left(F_{-W},-W, I_{t}\right)$ is also computed as a reference value $r$. Then, once we begin to match $I_{t}$ and $F_{u^{\prime}, v^{\prime}}$ for some $\left(u^{\prime}, v^{\prime}\right)$ depicted above, not only is $\operatorname{SRD}_{\tau}^{0, p}\left(F_{u^{\prime}, v^{\prime}}, I_{t}\right)$ computed, but a set of increasingly larger lower bounds $\operatorname{SRD}_{\tau}^{0, p}\left(F_{u^{\prime}, v^{\prime}}, I_{t}\right), \operatorname{SRD}_{\tau}^{1, p}\left(F_{u^{\prime}, v^{\prime}}, I_{t}\right)$, $\operatorname{SRD}_{\tau}^{2, p}\left(F_{u^{\prime}, v^{\prime}}, I_{t}\right), \ldots$ are also computed in turn if necessary. Once some $\operatorname{SRD}_{\tau}^{i, p}\left(F_{u^{\prime}, v^{\prime}}, I_{t}\right)(i=0,1, \ldots, n)$ is larger than the current reference value $r$, we have no need to compute all the other $\operatorname{SRD}_{\tau}^{j, p}\left(F_{u^{\prime}, v^{\prime}}, I_{t}\right)$ for $j=i+1, \ldots, n$, and the matching process between $I_{t}$ and $F_{u^{\prime}, v^{\prime}}$ can be terminated. We can then jump to another matching process between $I_{t}$ and the next image block of $F_{u^{\prime}, v^{\prime}}$ in the row-major order. This method can be treated as using a depth-first search procedure in visiting the search tree as shown in Fig. 3, and pruning the search branches once the computed error associated with the tree vertex is larger than the current reference value.

Recently, Chen et al. [7] extended the above method by exploiting the uniform cost search [34] in the tree instead of the depth-first search so that the search order is not fixed. First, the smallest value among all the errors of the highest level is found as

$$
\operatorname{SRD}_{\tau}^{0, p}\left(F_{u^{*}, v^{*}}, I_{t}\right)=\min (\text { the elements of } \boldsymbol{A})
$$

where $\boldsymbol{A}=\left\{\mathrm{SRD}_{\tau}^{0, p}\left(F_{-W,-W}, I_{t}\right), \mathrm{SRD}_{\tau}^{0, p}\left(F_{-W,-W+1}, I_{t}\right)\right.$, $\left.\ldots \operatorname{SRD}_{\tau}^{0, p}\left(F_{W, W}, I_{t}\right)\right\}$ is referred to as the active list, and $\left(u^{*}, v^{*}\right)$ is referred to as the temporary winner. Then, the error of the temporary winner in its next lower layer $\operatorname{SRD}_{\tau}^{1, p}\left(F_{u^{*}, v^{*}}, I_{t}\right)$ is computed. Next, the active list is updated by replacing $\operatorname{SRD}_{\tau}^{0, p}\left(F_{u^{*}, v^{*}}, I_{t}\right)$ with $\operatorname{SRD}_{\tau}^{1, p}\left(F_{u^{*}, v^{*}}, I_{t}\right)$ :

$$
\begin{gathered}
\boldsymbol{A} \leftarrow \boldsymbol{A} \cup\left\{\operatorname{SRD}_{\tau}^{1, p}\left(F_{u^{*}, v^{*}}, I_{t}\right)\right\} \backslash\left\{\operatorname{SRD}_{\tau}^{0, p}\left(F_{u^{*}, v^{*}}, I_{t}\right)\right\}, \\
\text { where " } \backslash " \text { is the set difference. }
\end{gathered}
$$

Then, the new minimal value among the elements in the new active list can be found as

$$
\operatorname{SRD}_{\tau}^{i, p}\left(F_{u_{1}^{*}, v_{1}^{*}}, I_{t}\right)=\min (\text { the elements of } \boldsymbol{A})
$$


where $i$ is now either 0 or 1 , and a new temporary winner $\left(u^{*}, v^{*}\right)$ is obtained. Repeat the above procedure of alternately updating the active list and finding the minimal value of the elements contained in it. Then, the minimal matching error can be found when $\left(u^{*}, v^{*}\right)$ reaches the lowest level. In general, this method can prune more unnecessary branches because the uniform cost search strategy is used.

Once the $p$-pyramids have been constructed for the template and image blocks using the method introduced in this paper, it can be incorporated into any of the search strategies introduced above in order to speed up the process of robust template matching. In this work, the search strategy developed in [7] is adopted because the experimental results in [7] show that better speedup performance can be obtained compared with other approaches for nonrobust template matching.

\section{B. Main Algorithm}

The algorithm of our approach using the uniform cost search for fast robust template matching is given in the following.

Step 1) Initially, set $\boldsymbol{A}=\left\{\mathrm{SRD}_{\tau}^{0, p}\left(F_{-W,-W}, I_{t}\right), \mathrm{SRD}_{\tau}^{0, p}\right.$ $\left.\left(F_{-W,-W+1}, I_{t}\right), \ldots, \operatorname{SRD}_{\tau}^{0, p}\left(F_{W, W}, I_{t}\right)\right\}$.

Step 2) Find $\left(u^{*}, v^{*}\right)$ such that $\operatorname{SRD}_{\tau}^{0, p}\left(F_{u^{*}, v^{*}}, I_{t}\right)$ is the minimum among all the elements in $\boldsymbol{A}$.

Step 3) $i \leftarrow 0$.

Step 4) While $(i \neq n)$

4.1) Compute $\operatorname{SRD}_{\tau}^{i+1, p}\left(F_{u^{*}, v^{*}}, I_{t}\right)$.

4.2) $\boldsymbol{A} \leftarrow \boldsymbol{A} \cup\left\{\operatorname{SRD}_{\tau}^{i+1, p}\left(F_{u^{*}, v^{*}}, I_{t}\right)\right\} \backslash\left\{\operatorname{SRD}_{\tau}^{i, p}\left(F_{u^{*}, v^{*}}, I_{t}\right)\right\}$.

4.3) Find $\left(u^{*}, v^{*}, j\right)$ such that $\operatorname{SRD}_{\tau}^{j, p}\left(F_{u^{*}, v^{*}}, I_{t}\right)$ is the minimum among all the elements in $\boldsymbol{A}$.

4.4) $i \leftarrow j$.

end While

Step 5) Output $\left(u^{*}, v^{*}\right)$.

The above algorithm applies the "uniform cost search" [34] to the tree illustrated in Fig. 3, which guarantees to find the global minimum solution as demonstrated in the following. When the algorithm goes to step 5, we know that $\operatorname{SRD}_{\tau}^{n, p}\left(F_{u^{*}, v^{*}}, I_{t}\right)$ is the minimum among all the elements contained in $\boldsymbol{A}$. In addition, from Theorem 1, the matching error computed for any two images on the level $n$ is not smaller than that for the other levels. Assume that $\boldsymbol{A}=\left\{\mathrm{SRD}_{\tau}^{n_{-W,-W}, p}\right.$ $\left(F_{-W,-W}, I_{t}\right), \mathrm{SRD}_{\tau}^{n_{-W,-W+1}, p}\left(F_{-W,-W+1}, I_{t}\right), \ldots, \mathrm{SRD}_{\tau}^{n_{W, W}, p}$ $\left.\left(F_{W, W}, I_{t}\right)\right\}$. Then, $\operatorname{SRD}_{\tau}^{n, p}\left(F_{i, j}, I_{t}\right) \geq \operatorname{SRD}_{\tau}^{n_{i, j}, p}\left(F_{i, j}, I_{t}\right)$ $\geq \operatorname{SRD}_{\tau}^{n, p}\left(F_{u^{*}, v^{*}}, I_{t}\right)$ for all $\operatorname{SRD}_{\tau}^{n_{i, j}, p}\left(F_{i, j}, I_{t}\right) \in \boldsymbol{A}$, or equivalently, for all $-W \leq i, j \leq W$, which shows that $\operatorname{SRD}_{\tau}^{n, p}\left(F_{u^{*}, v^{*}}, I_{t}\right)$ is the global minimal on the level $n$.

In fact, instead of from the highest to the lowest levels (i.e., from level 0 to level $n$ ), the search process can also be performed from an arbitrary middle level, say $m(0<m<n)$, to the lowest level $n$. This can simply be achieved by replacing each $\mathrm{SRD}_{\tau}^{0, p}$ with $\mathrm{SRD}_{\tau}^{m, p}$ in the Steps 1 and 2 of the main algorithm and further modifying Step 3 to be $i=m$. However, in our experience, it is better to select the starting level based on the ratio of outliers. In particular, we find that starting from a middle level (instead of the highest level) usually makes the process of robust template matching more efficient in practice. This is because the outliers contained in the template are included in the highest level image, although they may not be included in some middle level images. Some experimental results for the speedup versus different combinations of the starting levels and outlier ratios are shown in Section IV.

In practice, some standard methods [30], [38], [40] can be used to estimate an appropriate value for the parameter $\sigma$ in robust estimation. For example, the "median absolute deviation" scale estimate, which is related to the median of the absolute values of the residuals, is given by

$$
\hat{\sigma}=1.4826\left[1+\frac{5}{(n-p)}\right] \underset{i}{\operatorname{median}}\left|r_{i}\right|
$$

where the constant 1.4826 is a finite sample correction factor, $n$ is the size of the data set, $p$ is the dimension of the parameter vector, and $r_{i}$ is the residual error.

\section{EXPERIMENTAL RESULTS}

In this section, we present the results of three different experiments, including signal matching, face template matching, and motion estimation.

\section{A. Signal Matching}

In this experiment, we perform a simulation of searching a particular 1-D pattern along a 8192-point input signal, ranging from 0 to 255 , which is synthesized using a linear regression model. Four such input signals are used for this experiment, and one of them is shown in Fig. 4(a). We first randomly extract a 512-point partial segment, which is called the true identity signal, from an input signal. A 512-point test signal can then be generated by adding both Gaussian noise and some outliers to the true identity signal, as shown in Fig. 4(b)-(d), respectively. The outlier ratio (i.e., the ratio of the number of outlier points to the length of a test signal, 512) varies from 0 to 0.15 . Then, the test signal is used as a template and we try to find its matching segment in the input signal from which the test signal is extracted. In fact, this experiment simulates stereo matching (i.e., matching along a scan-line or epi-polar line) in computer vision [20]. Here, the SRD is used as the matching criterion. For each input signal and each outlier ratio, we first randomly generate 30 test signals and then find their matching segment in the input signal. The simple-truncation function (10) is used in this experiment as a robust error measure to suppress the effects of outliers. If the matching segment is not equal to its true identity signal, a miss occurs. Otherwise, a hit occurs. In this experiment, high average hit ratios ranging from $99.7 \%$ to $99.8 \%$ are achieved for all outlier ratios tested, which indicates that the M-estimator is very useful for suppressing the outlier effects. In the following, we focus on the main issue of this paper, the speedup of robust template matching, by comparing the efficiencies of our method with respect to those of the FS method.

First, we investigate the advantage of our method for the reduction of the major operations involved in the SRD computations. We define a robust operation to be the computation of the robust error measure $\rho(\cdot, \cdot)$, and computing $\rho(\cdot, \cdot) n$ times is therefore referred to as that $n$ robust operations are performed. 

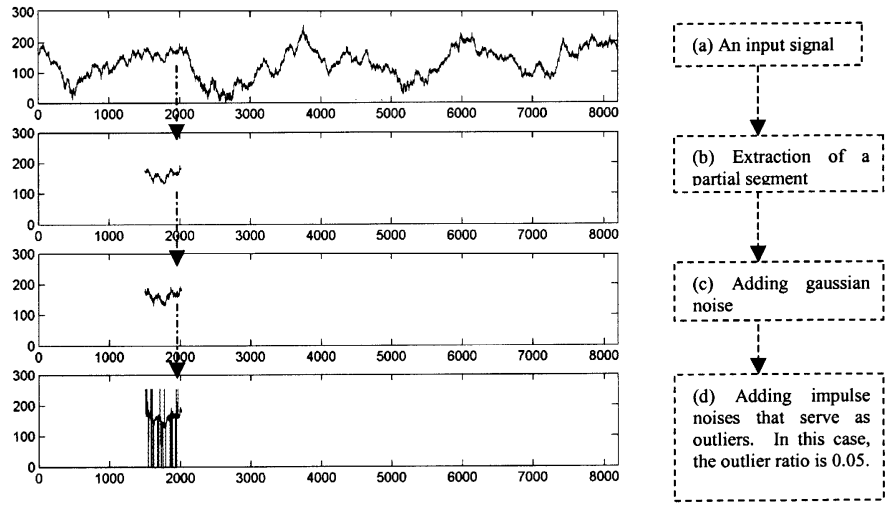

Fig. 4. (a) One of the synthetic input signals. (b)-(d) Process of generating a test signal from an input signal.

The robust operation is generally the most critical part for obtaining an SRD. The efficiency improvement with our method is evaluated by comparing the following two ratios: 1) the ratio of robust operations involved in our method to that in the FS method, and 2) the ratio of the execution time with our method to that with the FS method. These two ratios are referred to as the operation count ratio and the time consumption ratio, respectively. Evaluation of the efficiency improvement based on the operation count ratios is machine independent, but some additional computational overheads such as the construction of the $p$-pyramids and the switching among the search braches can not be reflected by the operation count ratios. On the other hand, evaluation based on the time consumption ratios includes all the overheads, but is machine dependent. In this experiment, both the operation count ratios and time consumption ratios are computed for evaluations and comparisons. In the setting of this experiment, there are a total of ten levels (level 0 to level 9) in the pyramid because the length of the test signal (512) is equal to $2^{9}$. Remember that our algorithm can start from any of the middle levels, as described in Section IV. Therefore, we also compare the speedup effect when the matching processes start from different initial levels in this experiment.

Since the simple truncation function is minimal 1-feasible, the 1-pyramid is constructed for robust template matching with our method. The operation count ratios using 1-pyramid are shown in Fig. 5(a). From Fig. 5(a), the operation count ratio varies overall from 0.1 to 0.48 when the outlier ratio varies from 0 to 0.15 if the middle value of the operation count ratios serves as a representative for each outlier ratio. This shows that our method can successively reduce the number of the major operations required for robust template matching, and the smaller the outlier ratio, the more reductions are achieved. An interesting phenomenon is that the best starting level (i.e., the starting level associated with the smallest operation count ratio) for each outlier ratio is a middle level. For example, when the outlier ratio lies in [0 0.02], the best starting level is 5 , whereas when the outlier ratios are increased to be within [0.04 0.08 and [ 0.10 .15$]$, the associated best starting levels become 6 and 7, respectively. The reason for this is that the outliers are easily to be included in the accumulation process for building a high level image in the pyramid, and thus, almost all the lower bounds with respect to a high level

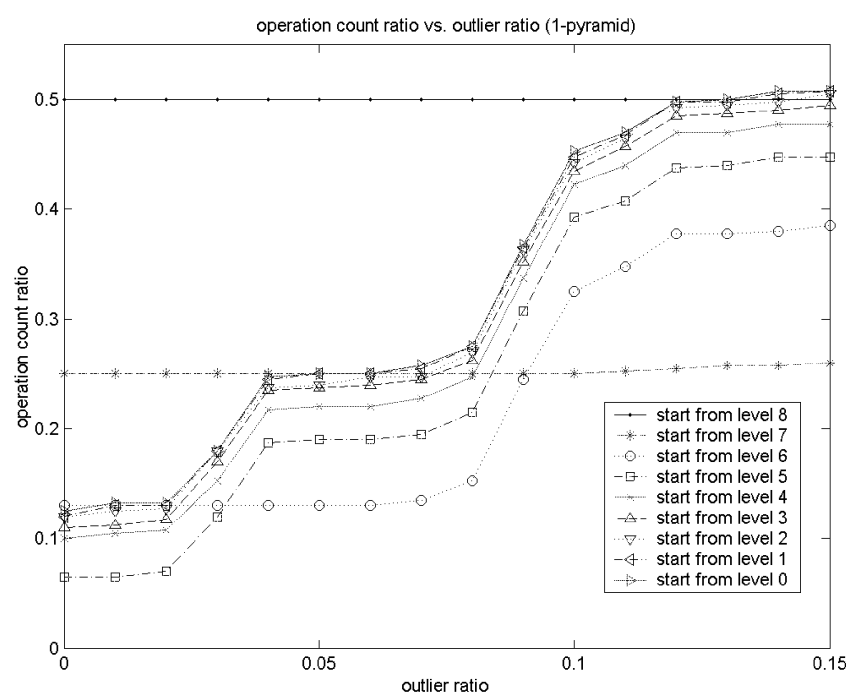

(a)

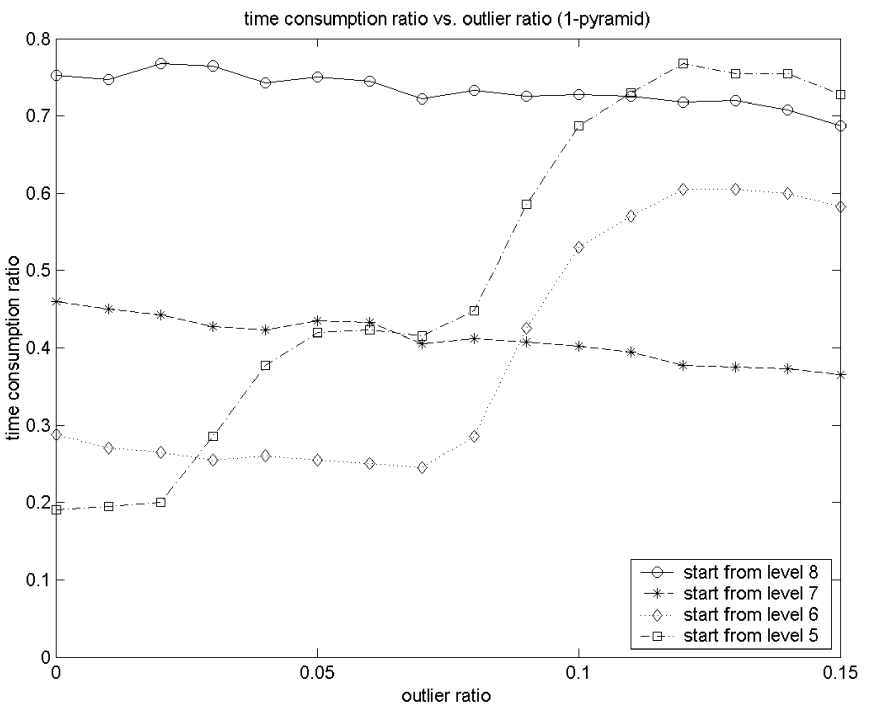

(b)

Fig. 5. Comparisons between our method and the FS method for robust template matching in the signal matching experiment. Note that simple truncation and the 1-pyramid are used in this experiment. (a) Operation count ratio versus outlier ratio. (b) Time consumption ratio versus outlier ratio.

image are required to be computed in the search process. On the other hand, when starting from a low level image, the lower bounds may not be tight enough to prune the search branches. Another interesting phenomenon is that the best starting level becomes lower as the outlier ratio increases.

Fig. 5(b) shows the time-consumption ratios taking into account not only the major operations but all the computational overheads such as pyramid constructions and controlling processes of search. The test was performed on a PC with the Visual $\mathrm{C}++$ language, and the middle levels, $5,6,7$, and 8 were used as the starting levels, respectively. From Fig. 5(b), the time consumption ratio varies overall from 0.4 to 0.6 when the outlier ratio varies from 0 to 0.15 , indicating that our method can also increase the efficiency of robust template matching in practice. Similarly, the best starting level becomes lower when the outlier ratio increases, and the best time-consumption ratio varies from 0.2 to 0.4 . Hence, if priori knowledge about the outlier 
TABLE I

COMParisons of Average Time-CONSUMPTION RATIO $(t c)$ AND Average OPERATION COUNT RATIOS (oc) OF OUR METHOD WHEN 1-PYRAMID, 2-PYRAMID, AND $\infty$-PYRAMID ARE USED

\begin{tabular}{|c|c|c|c|c|c|c|c|}
\hline & \multicolumn{2}{|c|}{ 1-pyramid } & \multicolumn{2}{|c|}{ 2-pyramid } & \multicolumn{2}{|c|}{$\infty$ - pyramid } \\
\hline & & $o c$ & $t c$ & $o c$ & $t c$ & $o c$ & $t c$ \\
\hline \multirow{4}{*}{ 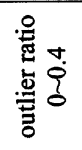 } & start from level 8 & 0.5 & 0.755 & 0.5 & 0.716 & 0.506 & 0.696 \\
\hline & start from level 7 & 0.25 & 0.441 & 0.256 & 0.436 & 0.291 & 0.468 \\
\hline & start from level 6 & 0.13 & 0.268 & 0.142 & 0.297 & 0.259 & 0.474 \\
\hline & start from level 5 & 0.102 & 0.250 & 0.139 & 0.331 & 0.314 & 0.629 \\
\hline \multirow{4}{*}{ 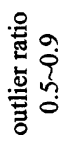 } & start from level 8 & 0.5 & 0.735 & 0.501 & 0.715 & 0.525 & 0.692 \\
\hline & start from level 7 & 0.25 & 0.419 & 0.269 & 0.443 & 0.392 & 0.595 \\
\hline & start from level 6 & 0.159 & 0.292 & 0.246 & 0.449 & 0.462 & 0.76 \\
\hline & start from level 5 & 0.22 & 0.458 & 0.309 & 0.619 & 0.536 & 0.951 \\
\hline \multirow{4}{*}{ 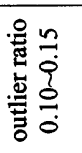 } & start from level 8 & 0.5 & 0.714 & 0.507 & 0.707 & 0.588 & 0.753 \\
\hline & start from level 7 & 0.255 & 0.381 & 0.353 & 0.541 & 0.588 & 0.828 \\
\hline & start from level 6 & 0.365 & 0.582 & 0.470 & 0.758 & 0.730 & 1.081 \\
\hline & start from level 5 & 0.429 & 0.737 & 0.533 & 0.904 & 0.796 & 1.234 \\
\hline
\end{tabular}

ratio of the template matching problem to be solved is given in advance, this can serve as a guideline to choose the best (or a better) starting level.

Notice that the simple truncation is minimal 1-feasible, and it is therefore also $p$-feasible for all $p \in[1 \infty]$. In the following, different $p$-pyramids (1-pyramid, 2-pyramid, and $\infty$-pyramid) are, respectively, constructed to compare their efficiencies when they are incorporated into our method. Their average operation count and time consumption ratios are summarized in Table I, showing that the speedup performance degrades when either the 2 -pyramid or the $\infty$-pyramid is used. This matches our claim above that the closer is $p$ to its minimal feasible value, the better speedup is achieved when the $p$-pyramid is used.

A summary of the above experimental results is given in what follows. First, the hit ratio is high when the technique of robust template matching is used, confirming that the SRD can suppress the affection of outliers. Second, from both the operation count and the time consumption ratios, our method is more efficient than the FS method. The amount of speedup achieved depends on many issues such as outlier ratios, starting levels, and the $p$-pyramid being used. In practice, the starting level should be selected according to the outlier ratio. If an estimation of the outlier is available in advance, the best starting level can be chosen according to the corresponding simulation results. As for which $p$-pyramid is suitable to be adopted in our method for a given robust error measure, it is suggested that the closer $p$ is to its minimal feasible value, the more speedup is achieved.

\section{B. Face Template Matching}

We perform face template matching experiments in a face-only database [27], which can be used for the application of finding a particular person in a database. We use 1000 images of 100 persons, where each person has ten images. Each image size is normalized to $64 \times 64$, as shown in Fig. 6(a). For each person, we randomly select one of his (or her) images for testing, with remaining nine for training. All test images are contaminated by pepper-and-salt noise that is used as outliers, with the outlier ratios varying from 0 to 0.15 . Fig. 6 (b) shows

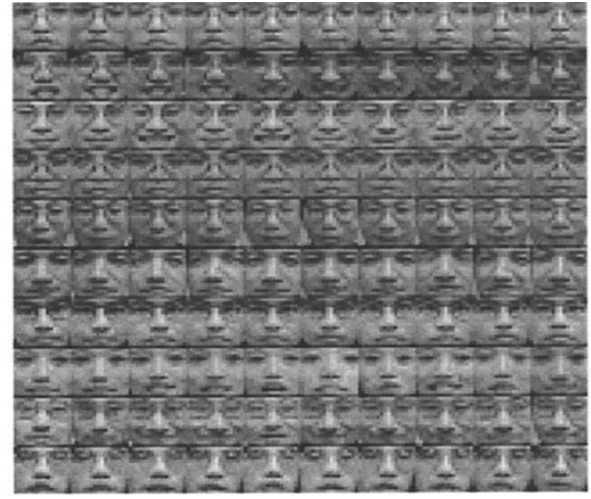

(a)

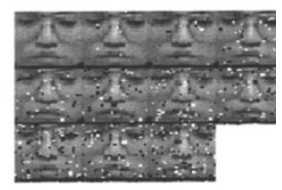

(b)
Fig. 6. (a) Part of a face-only database used in this paper, showing 100 images from ten people with ten images for each person. (b) Contaminated images of a person with different outlier ratios. From left to right and top to bottom, the outlier ratios are set from 0 to 0.1 .

the contaminated images of a person with different outlier ratios. Such an experimental setup is similar to that shown in [24], but in our case, the templates are polluted with outliers. Given a test image, we match it with the 900 images contained in the database and find the most similar one with the least sum of robust differences. The person with respect to the most similar image is then served as the recognized one. The matching experiment is performed for all of the 100 test images, and the average recognition rate (i.e., the hit ratio) is recorded.

Four different robust estimators (Huber's estimator, Tukey's estimator, Geman's and McClure's estimator, and the trimmed mean M-estimator) are used in this experiment. Based on (15), the parameter $\sigma$ used in these estimators was determined by performing several random matches to estimate median $_{i}\left|r_{i}\right|$ in advance. The associated 2-pyramids are constructed based on these robust estimators to speed up the corresponding robust template matching processes. First, we compare the recognition performances of template matching, using the above four robust estimators to that using the SSD criterion. Figs. 7(a), 8(a), 9(a), and 10(a) show the comparison results for Huber's estimator, Tukey's estimator, Geman's, and McClure's estimator and the trimmed mean M-estimator, respectively. From these figures, the hit ratios obtained using SRD are generally better than those obtained using SSD, no matter which estimators are used. In particular, the hit ratios obtained by using Tukey's estimator and Geman and McClure's estimators consistently perform better than those using SSD in all experiments, no matter which outlier ratios are tested. This observation also confirms that the M-estimator can deal with outliers better.

In the following, we present the speedup performances of our method. In particular, we focus on the time consumption ratios in this experiment. Since the pyramids of the images contained in the database are constructed offline for this application, the pyramid-construction time for the images contained the database is not included in the time consumption ratio in this experiment. However, note that the pyramid construction time of the test image has remained to be included in the computation of time consumption ratios. The time-consumption ratios of the above four robust estimators are shown in Figs. 7(b), 8(b), 9(b), and 10(b), respectively. From these figures, it can be seen that our method can increase the efficiencies for the face template 


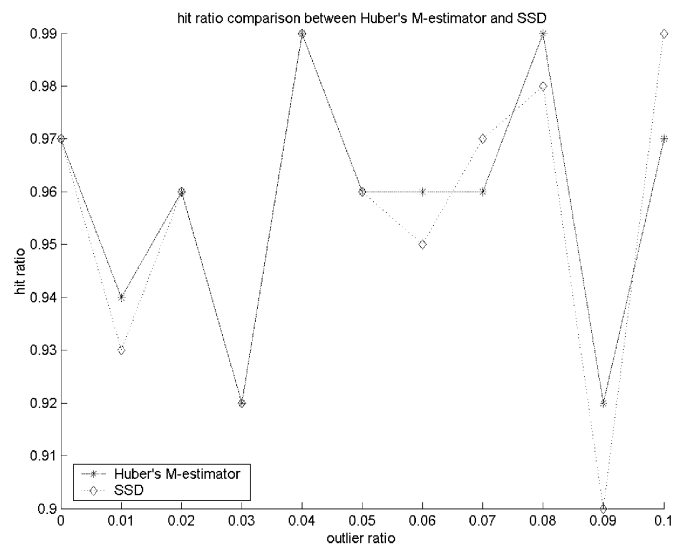

(a)

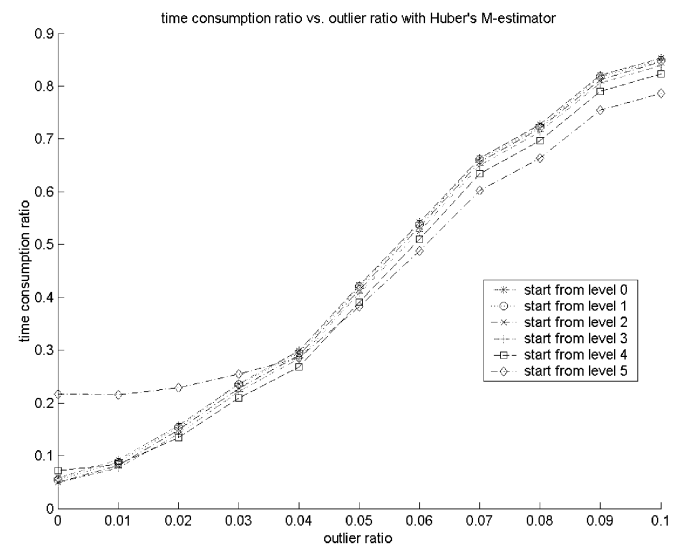

(b)

Fig. 7. Comparisons between the SSD and SRD using Huber's estimator. (a) Hit ratio versus outlier ratio. (b) Time consumption ratio versus outlier ratio.

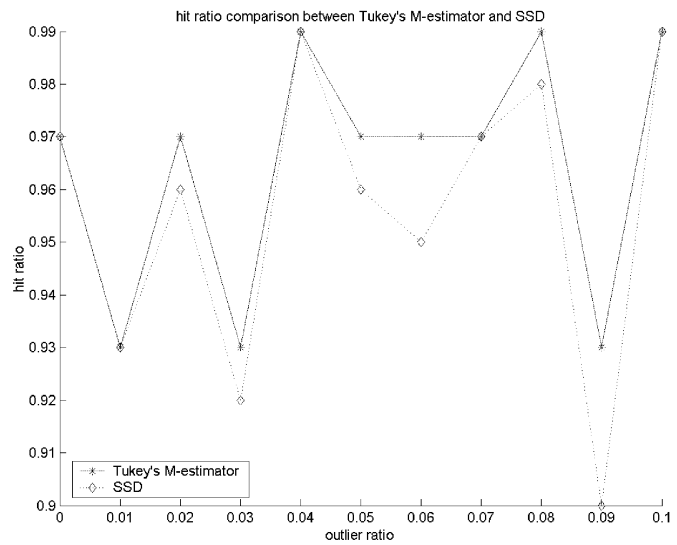

(a)

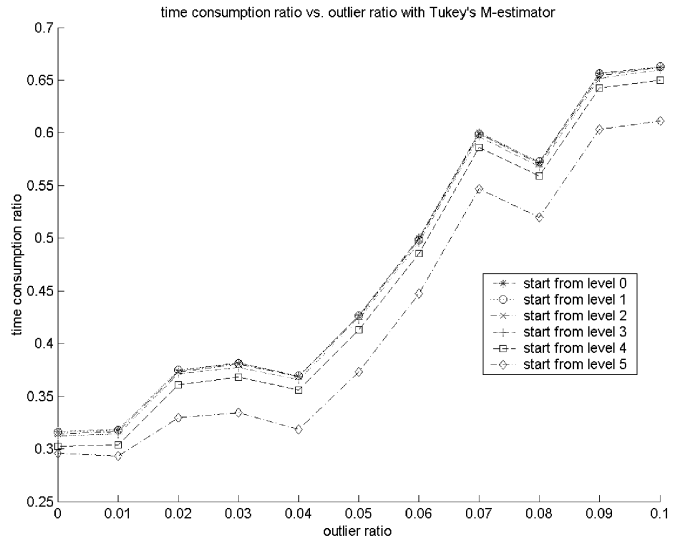

(b)

Fig. 8. Comparisons between the SSD and the SRD using Tukey's estimator. (a) Hit ratio versus outlier ratio. (b) Time consumption ratio versus outlier ratio.

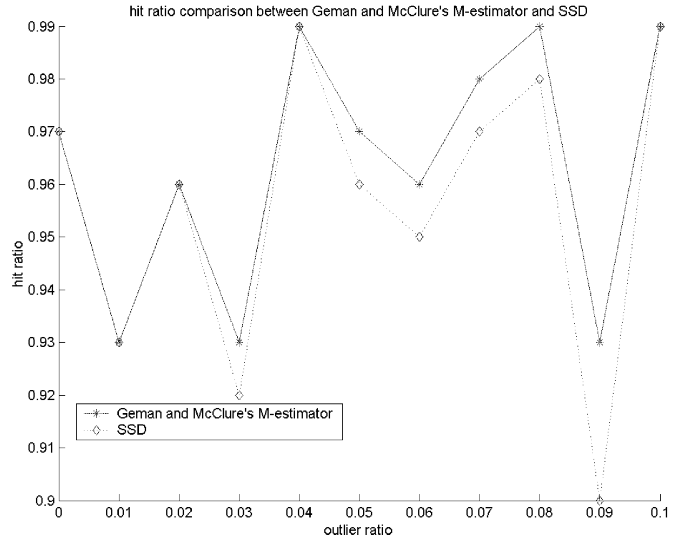

(a)

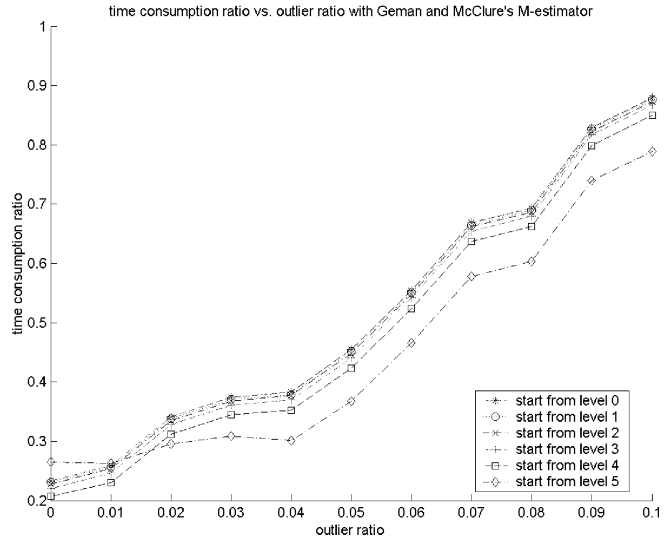

(b)

Fig. 9. Comparisons between the SSD and the SRD using Geman's and McClure's estimator. (a) Hit ratio versus outlier ratio. (b) Time consumption ratio versus outlier ratio.

matching for different kinds of robust estimators. In essence, the smaller is the outlier ratio, the better speedup is achieved. In particular, the speed performances depend on what kind of robust estimators are used. For example, the speedup performances are better when Huber's estimator, Tukey's estimator, and the trimmed mean M-estimator are used, than that when Geman's and McClure's estimator is used. A possible reason for this is that the lower bounds derived for the former estimators are tighter than those for the latter.

\section{Motion Estimation}

In the last experiment, we use our method for robust motion estimation in a sequence of images. Each image in the sequence is segmented into a set of blocks, and we try to find the motion vector for each block. Assume that one image in the sequence is polluted with outliers, and the robust template matching technique is used for motion estimation when outliers occur. The salesman image sequence is used as a test sequence where each frame is of size 352 


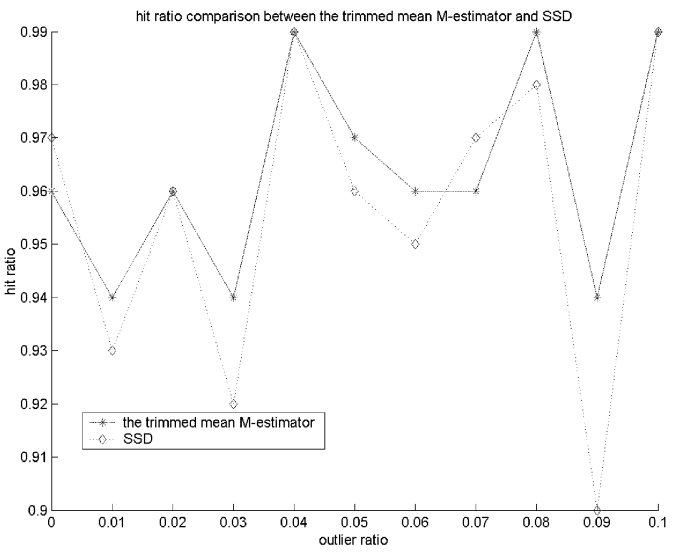

(a)

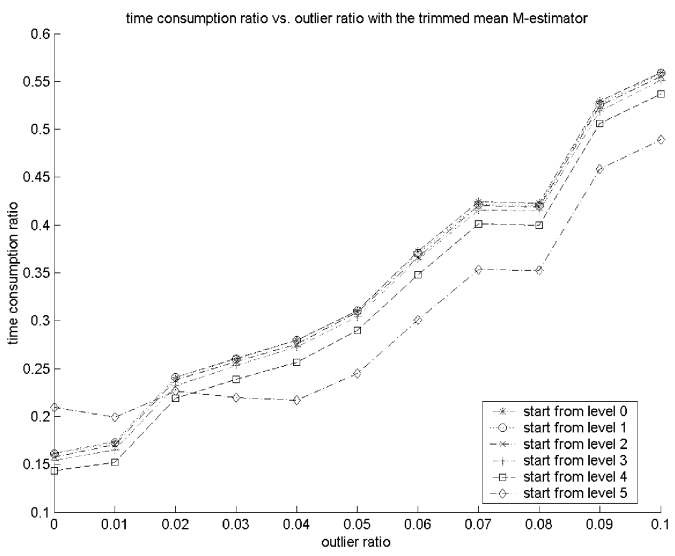

(b)

Fig. 10. Comparisons between the SSD and the SRD using the trimmed mean M-estimator. (a) Hit ratio versus outlier ratio. (b) Time consumption ratio versus outlier ratio.

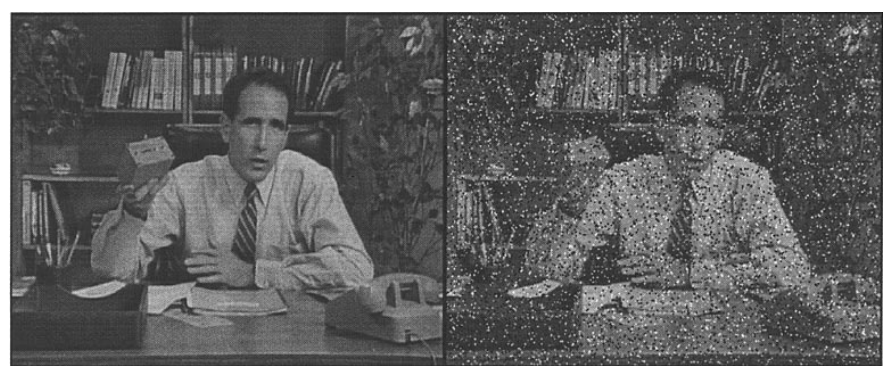

(a)

(b)

Fig. 11. Example of a pair of two consecutive frames for motion estimation. (a) Previous frame. (b) Current frame. Notice that the outlier ratio in (b) is $10 \%$

$\times 288$. Two different block sizes $(16 \times 16$ and $32 \times 32)$ and their corresponding search ranges (about twice as large as the block size $[-16,16] \times[-16,16]$ and $[-32,32] \times[-32,32])$ are tested in this experiment. Given a pair of two consecutive frames, where one is polluted as shown in Fig. 11, we use the simple truncation (10) as the robust error measure for robust template matching to find the motion vectors. The time-consumption ratios (that consider all the computational overheads) and the operation count ratios are shown in Fig. 12, respectively. The experimental results show that our method can also improve the efficiency for robust motion estimation.

\section{DISCUSSION}

From the experimental results, the efficiency improvement of our method improves if the outlier ratio gets smaller. In fact, the time-consumption ratio may exceed a value of one in our experiment when the outlier ratio is too large, which means that our method cannot speed up the matching process in this case. However, since the estimation problem is itself more difficult to solve accurately when the outlier ratio is too large, our method can be treated as dealing with common cases of robust template matching when M-estimators are used.

One possible means to allow large outlier ratios is to make the lower bounds tighter. In fact, the set of ascending lower bounds (accompanied with the minimal feasible value and the $p$-pyramid structure) derived in this paper is generally available for all nondecreasing robust error measures. Improvement is thus possible by tightening the lower bounds for a particular error measure, although the improved set of lower bounds may not be able to be used for other error measures. To achieve this goal, we can take advantage of the specific mathematical form of each of the error measures and derive new theoretical bounds and pyramid structures. Such an improvement remains an open problem and to be investigated in the future.

Our main algorithm was presented to tackle the matching problem with translation. When dealing with a problem with rotation or scaling, a useful strategy is to generate a set of templates by rotating and scaling the pattern to be matched in advance [21], [37]. In this case, a $p$-pyramid can be produced for each template and our algorithm can then be applied for speeding up the matching process with these templates. Such a pretransforming/prestoring process is suitable for applications where offline processing is allowed, such as the face-matching task shown in the experimental results of this paper. Another way for dealing with rotation (or illumination variation) is to use rotationally invariant (or lighting-invariant) features such as moment invariants [10]. By this way, the templates need to be preprocessed to extract their invariant feature vectors. To our best knowledge, however, there is no analysis about the sensitivities of the invariant vectors to outliers, and thus it is possible that they are not robust enough for matching with outliers. Nevertheless, when the noise model is Gaussian and a common SSD measure is applied for matching the feature vectors, our method can also be used to speed up the matching process by building the 2-pyramids associated with the invariant feature vectors in advance. ${ }^{6}$ In addition, another widely used way for dealing with illumination variation is to perform histogram equalization [18].

Another issue from the experimental results is that the best starting level becomes lower as the outlier ratio gets smaller. How to determine the best (or a better) starting level in an analytic way when the outlier ratio and a particular robust error measure are given also remains an open problem. In the meantime, the best starting level can be determined through a simulation process by taking some training examples from the problem to be solved.

${ }^{6}$ Note that extraction of moment invariants can itself be speeded up with some other fast algorithms [9]. 


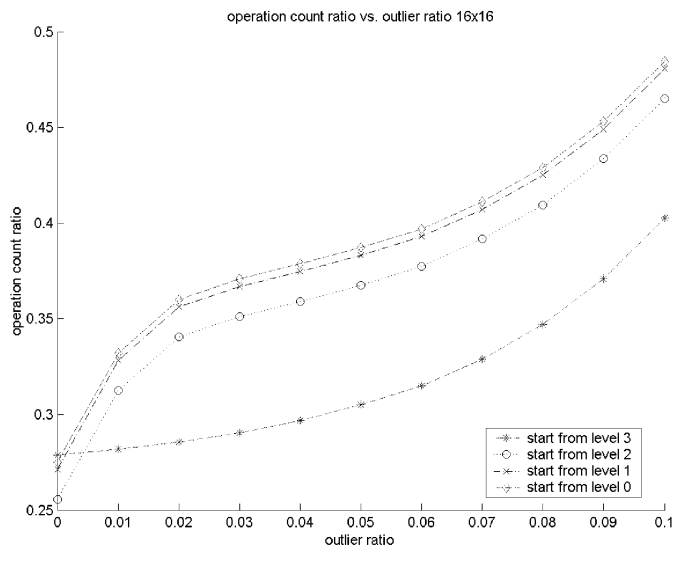

(a)

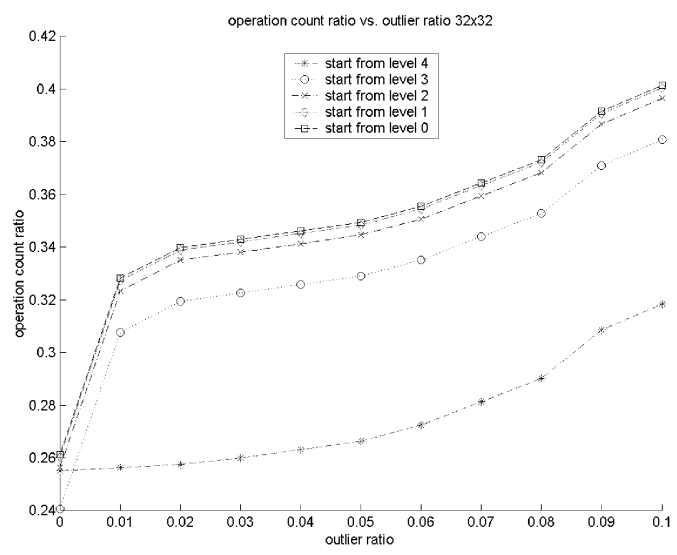

(c)

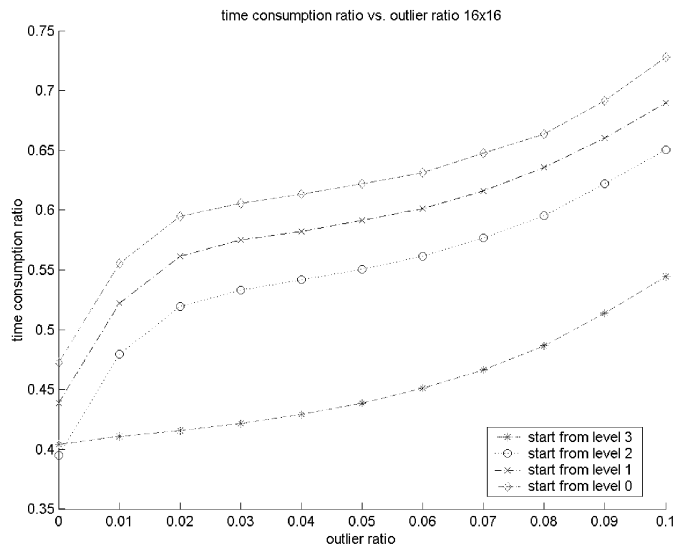

(b)

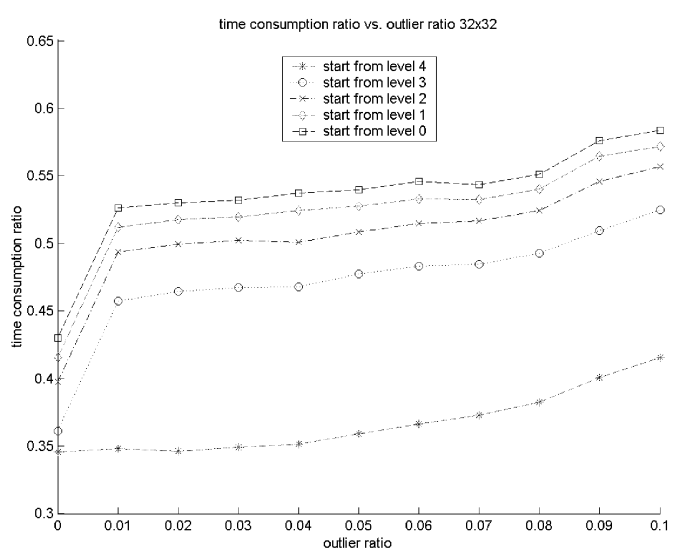

(d)

Fig. 12. (a) Operation count ratio versus outlier ratio when the block size is $16 \times 16$. (b) Time-consumption ratio versus outlier ratio when the block size is $16 \times$ 16. (c) Operation count ratio versus outlier ratio when the block size is $32 \times 32$. (d) Time-consumption ratio versus outlier ratio when the block size is $32 \times 32$.

\section{CONCLUSIONS}

In this paper, we have developed a systematic method that can be used to speed up the process of robust template matching when the error function is nondecreasing. We proposed the $p$-feasible related theory in Section II, which serves as a basis for constructing a hierarchical structure, the $p$-pyramid. This pyramid can then be used to generate a set of ascending lower bounds of the minimal matching error. As long as the error function is nondecreasing, there exists a $p$ making this function $p$-feasible. We have also shown that the smaller is $p$, the more efficient is the associated fast algorithm. In addition, many commonly used nondecreasing robust estimators are minimal 2-feasible or minimal 1-feasible, as shown in Section II-E.

By exploiting the property that the computations of the lower bounds generated with our method are more efficient than the computation of the matching errors directly, some fast search strategies can thus be used to speed up the matching process, as shown in Section III-A. In this paper, the uniform cost search strategy is adopted, and the experimental results show that our method can successfully increase the matching efficiencies when outliers exist.

A characteristic of our method is that it is easily generalized as not finding only the site with the minimal matching error but a series of sites having the $k$ minimal matching errors. To compute the $k$ minimal matching errors, we do not need to perform our algorithm $k$ times but continuously execute the best-search procedure shown in Section III-B until $k$ leaf nodes have been computed. Hence, the robust $k$-nearest neighbor problem can be solved in a unique procedure. Moreover, our method can also be used for improving the matching efficiency if a suboptimal or approximate solution is allowed to be generated instead of the solution with the minimal matching error. This can be achieved by using a middle level, instead of the lowest level, as being the level containing the leaf nodes.

To our knowledge, this is the first approach proposed to speed up the process of robust template matching. The method proposed in this paper gives a generally useful scenario for solving this type of problem. Our method also provides some significant research directions that can be further studied, including the investigations of tighter lower bounds, the generalizations to include the error measures that are not nondecreasing, and the analytical decisions for best starting levels.

\section{APPENDIX A PROOF OF PROPERTY 2}

Proof: If a robust error measure $\tau(\cdot)$ is $p$-feasible, to prove that $\tau(\cdot)$ is also $q$-feasible for all $q \in[p \infty]$ is equivalent to proving that $\tau(\cdot)$ satisfies the following inequality for all $q \in$ $[p \infty]$ :

$$
\forall a_{1}, a_{2} \in R^{+} \cup\{0\}, \quad \tau\left(a_{1}\right)+\tau\left(a_{2}\right) \geq \tau\left(\|\boldsymbol{a}\|_{q}\right) .
$$


We first introduce three lemmas before proving (A.1) for all $q \in\left[\begin{array}{ll}p & \infty\end{array}\right]$. Assume that $\mathbf{x}=\left[\begin{array}{ll}x_{1} & x_{2}\end{array}\right]^{t} \in R^{2}$. In addition, we denote $\mathbf{B}_{\|.\|_{\alpha}}=\left\{\mathbf{x} \mid\|\mathbf{x}\|_{\alpha} \leq 1\right\}$, to be the unit ball with respect to the $L_{\alpha}$ norm.

Lemma 1: If $\alpha \in[1 \infty]$ and $\mathbf{x} \in \mathbf{B}_{\|\cdot\|_{\alpha}}$, then $1 \geq\left|x_{1}\right| \geq 0$ and $1 \geq\left|x_{2}\right| \geq 0$.

Proof: The proof is trivial.

Lemma 2: If $\alpha, \beta \in[1 \infty]$ and $\beta \geq \alpha$, then $\mathbf{B}_{\|.\|_{\alpha}} \subseteq \mathbf{B}_{\|.\|_{\beta}}$.

Proof: We prove this lemma by considering the following two cases: $\alpha<\beta$ and $\alpha=\beta$.

Case $1(\alpha<\beta): \forall \mathbf{x} \in \mathbf{B}_{\|\cdot\|_{\alpha}}$

$$
\begin{aligned}
\left(\left|x_{1}\right|^{\alpha}+\left|x_{2}\right|^{\alpha}\right)^{1 / \alpha} \leq 1 & \\
\Rightarrow 0 & \leq\left(\left|x_{1}\right|^{\alpha}+\left|x_{2}\right|^{\alpha}\right) \leq 1 \\
\Rightarrow 0 & \leq\left(\left|x_{1}\right|^{\beta}+\left|x_{2}\right|^{\beta}\right) \\
& =\left(\left|x_{1}\right|^{\beta-\alpha}\left|x_{1}\right|^{\alpha}+\left.\left|x_{2}\right|^{\beta-\alpha}|| x_{2}\right|^{\alpha}\right) \\
& \leq\left(\left|x_{1}\right|^{\alpha}+\left|x_{2}\right|^{\alpha}\right) \leq 1 \\
\Rightarrow 0 & \leq\left(\left|x_{1}\right|^{\beta}+\left|x_{2}\right|^{\beta}\right)^{1 / \beta} \leq 1 \\
\Rightarrow \mathbf{x} & \in \mathbf{B}_{\|.\|_{\beta}} .
\end{aligned}
$$

Case $2(\alpha=\beta)$ : This case is trivial.

From the above two cases, if $\alpha, \beta \in[1 \infty]$, and $\beta \geq \alpha$, then $\mathbf{B}_{\|\cdot\|_{\alpha}} \subseteq \mathbf{B}_{\|\cdot\|_{\beta}}$.

Lemma 3: If $\alpha, \beta \in[1 \infty]$, and $\mathbf{B}_{\|\cdot\|_{\alpha}} \subset \mathbf{B}_{\|\cdot\|_{\beta}}$, then $\forall \mathbf{x}$, $\|\mathbf{x}\|_{\alpha} \geq\|\mathbf{x}\|_{\beta}$.

Proof: See [17, p. 282].

From Lemmas 2 and 3, we know that if $\alpha, \beta \in[1 \infty]$ and $\alpha \leq \beta$, then $\forall \mathbf{x},\|\mathbf{x}\|_{\alpha} \geq\|\mathbf{x}\|_{\beta}$. Since $\tau(\cdot)$ is $p$-feasible and $\|\mathbf{x}\|_{p} \geq\|\mathbf{x}\|_{q}$ for all $q \in[p \infty]$, then (A.1) holds for all $q \in$ $[p \infty]$. Hence, $\tau(\cdot)$ is also $q$-feasible for all $q \in[p \infty]$.

\section{APPENDIX B}

\section{PROOF OF THEOREM 1}

Proof: Given a robust error measure $\tau(\cdot)$ that is $p$-feasible for all $m \in\{0,1, \ldots, n-1\}$

$$
\begin{aligned}
\mathrm{SRD}_{\tau}^{m+1, p}\left(I_{1}, I_{2}\right) & \sum_{0 \leq i, j<2^{m+1}} \tau\left(\left|I_{1}^{m+1, p}(i, j)-I_{2}^{m+1, p}(i, j)\right|\right) \\
= & \sum_{0 \leq i, j<2^{m}}\left\{\tau\left(\left|I_{1}^{m+1, p}(2 i, 2 j)-I_{2}^{m+1, p}(2 i, 2 j)\right|\right)\right. \\
& +\tau\left(\left|I_{1}^{m+1, p}(2 i, 2 j+1)-I_{2}^{m+1, p}(2 i, 2 j+1)\right|\right) \\
& +\tau\left(\left|I_{1}^{m+1, p}(2 i+1,2 j)-I_{2}^{m+1, p}(2 i+1,2 j)\right|\right) \\
& +\tau\left(\mid I_{1}^{m+1, p}(2 i+1,2 j+1)\right. \\
& \left.\left.-I_{2}^{m+1, p}(2 i+1,2 j+1) \mid\right)\right\} \\
\geq & \sum_{0 \leq i, j<2^{m}}\left\{\tau \left(\left(\left|I_{1}^{m+1, p}(2 i, 2 j)-I_{2}^{m+1, p}(2 i, 2 j)\right|^{p}\right.\right.\right. \\
& \left.\left.+\left|I_{1}^{m+1, p}(2 i, 2 j+1)-I_{2}^{m+1, p}(2 i, 2 j+1)\right|^{p}\right)^{1 / p}\right) \\
& +\tau\left(\left(\left|I_{1}^{m+1, p}(2 i+1,2 j)-I_{2}^{m+1, p}(2 i+1,2 j)\right|^{p}\right.\right.
\end{aligned}
$$

$$
\begin{aligned}
& +\mid I_{1}^{m+1, p}(2 i+1,2 j+1) \\
& \left.\left.\left.\quad-\left.I_{2}^{m+1, p}(2 i+1,2 j+1)\right|^{p}\right)^{1 / p}\right)\right\} \\
\geq & \sum_{0 \leq i, j<2^{m}}\left\{\tau \left(\left(\left|I_{1}^{m+1, p}(2 i, 2 j)-I_{2}^{m+1, p}(2 i, 2 j)\right|^{p}\right.\right.\right. \\
& +\left|I_{1}^{m+1, p}(2 i, 2 j+1)-I_{2}^{m+1, p}(2 i, 2 j+1)\right|^{p} \\
& +\left|I_{1}^{m+1, p}(2 i+1,2 j)-I_{2}^{m+1, p}(2 i+1,2 j)\right|^{p} \\
& +\mid I_{1}^{m+1, p}(2 i+1,2 j+1) \\
& \left.\left.\left.-\left.I_{2}^{m+1, p}(2 i+1,2 j+1)\right|^{p}\right)^{1 / p}\right)\right\} \\
\geq & \sum_{0 \leq i, j<2^{m}}\left\{\tau \left(\mid\left(I_{1}^{m+1, p}(2 i, 2 j)^{p}+I_{1}^{m+1, p}(2 i, 2 j+1)^{p}\right.\right.\right. \\
& \left.+I_{1}^{m+1, p}(2 i+1,2 j)^{p}+I_{1}^{m+1, p}(2 i+1,2 j+1)^{p}\right)^{1 / p} \\
& -\left(I_{2}^{m+1, p}(2 i, 2 j)^{p}+I_{2}^{m+1, p}(2 i, 2 j+1)^{p}\right. \\
& +I_{2}^{m+1, p}(2 i+1,2 j)^{p} \\
& \left.\left.\left.+I_{2}^{m+1, p}(2 i+1,2 j+1)^{p}\right)^{1 / p} \mid\right)\right\} \\
= & \operatorname{SRD}_{\tau}^{m, p}\left(I_{1}, I_{2}\right)
\end{aligned}
$$

where the first two inequalities are based on constraint (4), and the last inequality is derived from both the triangle inequality ${ }^{7}$ and the fact that $\tau(\cdot)$ is nondecreasing.

\section{APPENDIX C \\ PROOF OF PROPERTY 4}

Assume that $\tau(\cdot)$ belongs to $H_{\rho_{1}}$. To prove that $\tau(\cdot)$ is minimal 2-feasible is equivalent to showing that $\tau(\cdot)$ is 2-feasible but not 1-feasible. Since it is trivial that $\tau(\cdot)$ is both nondecreasing and not 1-feasible, we focus on the proof of the fact that $\tau\left(a_{1}\right)+\tau\left(a_{2}\right) \geq \tau\left(\|\mathbf{a}\|_{2}\right)$ for all $a_{1}, a_{2} \in R^{+} \cup\{0\}$. Without loss of generality, assume that $0 \leq a_{2} \leq a_{1} \leq\|\mathbf{a}\|_{2}$, and consider the following four cases:

- Case $1\left(\sigma \geq\|\mathbf{a}\|_{2} \geq a_{1} \geq a_{2} \geq 0\right)$ :

$$
\begin{aligned}
\rho_{1}\left(a_{1}, \sigma\right)+\rho_{1}\left(a_{2}, \sigma\right)- & \rho_{1}\left(\|\mathbf{a}\|_{2}, \sigma\right) \\
& =\frac{a_{1}^{2}}{2}+\frac{a_{2}^{2}}{2}-\frac{a_{1}^{2}+a_{2}^{2}}{2}=0 .
\end{aligned}
$$

- Case $2\left(\|\mathbf{a}\|_{2} \geq \sigma \geq a_{1} \geq a_{2} \geq 0\right)$ :

$$
\begin{aligned}
\rho_{1}\left(a_{1}, \sigma\right)+\rho_{1}\left(a_{2}, \sigma\right) & -\rho_{1}\left(\|\mathbf{a}\|_{2}, \sigma\right) \\
= & \frac{a_{1}^{2}}{2}+\frac{a_{2}^{2}}{2}-\sigma \sqrt{a_{1}^{2}+a_{2}^{2}}+\frac{\sigma^{2}}{2} .
\end{aligned}
$$

Since

$$
\frac{\left(a_{1}^{2}+a_{2}^{2}\right)+\sigma^{2}}{2} \geq \sigma \sqrt{a_{1}^{2}+a_{2}^{2}} \geq 0
$$

according to the arithmetic-geometric inequality, (C.2) $\geq 0$.

${ }^{7}$ Given two vectors $\mathbf{a}$ and $\mathbf{b},\|\mathbf{a}-\mathbf{b}\|_{p} \geq\left|\|\mathbf{a}\|_{p}-\|\mathbf{b}\|_{p}\right|$. 
- Case $3\left(\|\mathbf{a}\|_{2} \geq a_{1} \geq \sigma \geq a_{2} \geq 0\right)$ :

$$
\begin{aligned}
\rho_{1}\left(a_{1}, \sigma\right)+\rho_{1}\left(a_{2}, \sigma\right)- & \rho_{1}\left(\|\mathbf{a}\|_{2}, \sigma\right) \\
& =\sigma a_{1}+\frac{a_{2}^{2}}{2}-\sigma \sqrt{a_{1}^{2}+a_{2}^{2}} .
\end{aligned}
$$

Since

$$
\begin{gathered}
\left(\sigma a_{1}+\frac{a_{2}^{2}}{2}\right)^{2}-\left(\sigma \sqrt{a_{1}^{2}+a_{2}^{2}}\right)^{2} \\
=\sigma a_{1} a_{2}^{2}+\frac{a_{2}^{4}}{4}-\sigma^{2} a_{2}^{2} \\
=a_{2}^{2}\left(\sigma\left(a_{1}-\sigma\right)+\frac{a_{2}^{2}}{4}\right) \geq 0
\end{gathered}
$$

and both two terms $\sigma a_{1}+\left(a_{2}^{2} / 2\right)$ and $\sigma \sqrt{a_{1}^{2}+a_{2}^{2}}$ are non-negative, (C.3) $\geq 0$.

- Case $4\left(\mid \mathbf{a} \|_{2} \geq a_{1} \geq a_{2} \geq \sigma>0\right)$ :

$$
\begin{aligned}
\rho_{1}\left(a_{1}, \sigma\right)+\rho_{1}\left(a_{2}, \sigma\right)-\rho_{1}\left(\|\mathbf{a}\|_{2}, \sigma\right) \\
=\sigma\left(a_{1}+a_{2}-\sqrt{a_{1}^{2}+a_{2}^{2}}-\frac{\sigma}{2}\right) .
\end{aligned}
$$

Since

$$
\begin{aligned}
& \left(a_{1}+a_{2}-\frac{\sigma}{2}\right)^{2}-\left(\sqrt{a_{1}^{2}+a_{2}^{2}}\right)^{2} \\
& =2 a_{1} a_{2}-\sigma a_{1}-\sigma a_{2}+\frac{\sigma^{2}}{4} \\
& =a_{1}\left(a_{2}-\sigma\right)+a_{2}\left(a_{1}-\sigma\right)+\frac{\sigma^{2}}{4}>0
\end{aligned}
$$

and both two terms $a_{1}+a_{2}-(\sigma / 2)$ and $\sqrt{a_{1}^{2}+a_{2}^{2}}$ are nonnegative, then (C.4) $\geq 0$.

The union of the above four cases shows that $\tau\left(a_{1}\right)+\tau\left(a_{2}\right) \geq$ $\tau\left(\|\mathbf{a}\|_{2}\right)$ for all $a_{1}, a_{2} \in R^{+} \cup\{0\}$.

\section{ACKNOWLEDGMENT}

The authors would like to thank the anonymous reviewers for their helpful and constructive comments.

\section{REFERENCES}

[1] M. Bierling, "Displacement estimation by hierarchical block-matching," in Proc. SPIE Conf. Visual Commun. Image Process., vol. 1001, 1988, pp. $942-951$.

[2] V. Barnett and T. Lewis, Outliers in Statistical Data. New York: Wiley, 1994.

[3] M. J. Black and P. Anandan, "The robust estimation of multiple motions: Parametric and piecewise-smooth flow fields," Comput. Vision Image Understanding, vol. 63, pp. 75-104, 1996.

[4] M. J. Black and A. Rangarajan, "On the unification of line processes, outlier rejection, and robust statistics with applications in early vision," Int. J. Comput. Vision, vol. 19, pp. 57-91, 1996.

[5] A. Can, C. V. Stewart, and B. Roysam, "Robust hierarchical algorithm for constructing a mosaic from images of the curved human retina," in Proc. IEEE Conf. Comput. Vision Pattern Recogn., 1999, pp. 286-292.

[6] J.-H. Chen, C.-S. Chen, and Y.-S. Chen, "Fast method for robust template matching," Institute of Information Science, Academia Sinica, Taipei, Taiwan, R.O.C., Tech. Rep. TR-IIS-01-015, 2001.

[7] Y.-S. Chen, Y.-P. Hung, and C.-S. Fuh, "Fast block matching algorithm based on the winner-update strategy," IEEE Trans. Image Processing, vol. 10, pp. 1212-1222, Aug. 2001.

[8] K. H. K. Chow and M. L. Liou, "Genetic motion search algorithm for video compression," IEEE Trans. Circuit Syst. Video Technol., vol. 3, pp. 440-445, Dec. 1993.
[9] J. Flusser, "Refined moment calculation using image block representation," IEEE Trans. Image Processing, vol. 11, pp. 1977-1978, Nov. 2000

[10] J. Flusser and B. Zitová, "Combined invariants to linear filtering and rotation,” Int. J. Pattern Recogn. Artificial Intell., vol. 13, pp. 1123-1136, 1999.

[11] H. Frigui and R. Krishnapuram, "A robust competitive clustering algorithm with applications in computer vision," IEEE Trans. Pattern Anal. Machine Intell., to be published.

[12] S. Geman and D. E. McClure, "Statistical methods for tomographic image reconstruction," Bull. Int. Statist. Inst., vol. 52, pp. 5-21, 1987.

[13] M. Ghanbari, "The cross-search algorithm for motion estimation," IEEE Trans. Commun., vol. 38, pp. 950-953, July 1990.

[14] R. M. Haralick et al., "Pose estimation from corresponding point data," IEEE Trans. Syst., Man, Cybern., vol. 19, pp. 1426-1446, Nov./Dec. 1989.

[15] H.-C. Huang, Y.-P. Hung, and W.-L. Hwang, "Adaptive early jump-out technique for fast motion estimation in video coding," CVGIP: Graph. Models Image Process., vol. 59, pp. 388-394, 1997.

[16] P. J. Huber, Robust Statistics. New York: Wiley, 1981.

[17] R. A. Horn and C. R. Johnson, Matrix Analysis. Cambridge, U.K.: Cambridge Univ. Press, 1985.

[18] K. Jonsson, J. Kittler, Y. P. Li, and J. Matas, "Support vector machines for face authentication," Image Vision Comput., to be published.

[19] K. Kawamura et al., "Object recognition using local EGI and 3D models with M-estimators," in Proc. IEEE Int. Conf. Multisensor Fusion Integration Intell. Syst., 1999, pp. 80-86.

[20] R. Klette, K. Schluns, and A. Koschan, Computer Vision Three-Dimensional Data from Images. $\quad$ New York: Springer, 1998, ch. 4.

[21] D. Keysers, W. Macherey, J. Dahmen, and H. Ney, "Learning of variability for invariant statistical pattern recognition," in Proc. 12th Eur. Conf. Machine Learning, Freiburg, Germany, 2001, pp. 263-275.

[22] T. Koga et al., "Motion compensated interframe coding for video conference," in Proc. Nat. Telecommun. Conf., 1981, pp. G5.3.1-G5.3.5.

[23] S.-H. Lai, "Robust image matching under partial occlusion and spatially varying illumination change," Comput. Vision Image Understanding, vol. 78, pp. 84-98, 2000.

[24] S. Lawrence, C. L. Giles, A. -C. Tsoi, and A. Back, "Face recognition: A convolutional neural network approach," IEEE Trans. Neural Networks, vol. 8, pp. 98-113, Jan. 1997.

[25] C.-H. Lee and L.-H. Chen, "A fast motion estimation algorithm based on the block sum pyramid," IEEE Trans. Image Processing, vol. 6, pp. 1587-1591, Oct. 1997.

[26] W. Li and E. Salari, "Successive elimination algorithm for motion estimation," IEEE Trans. Image Processing, vol. 4, pp. 105-107, Jan. 1995.

[27] H.-Y. M. Liao, C.-C. Han, and G.-J. Yu, "Face+hair+shoulders +blackground $\neq$ face," Inst. Inform. Sci., Academia Sinica, Taipei, Taiwan, R.O.C., Tech. Rep. TR-IIS-97-007, 1997.

[28] C.-H. Lin and J.-L. Wu, "A lightweight genetic block-matching algorithm for video coding," IEEE Trans. Circuit Syst. Video Technol., vol. 8, pp. 386-392, Aug. 1998.

[29] L.-K. Liu and E. Feig, "A block-based gradient descent search algorithm for block motion estimation in video coding," IEEE Trans. Circuit Syst. Video Technol., vol. 6, pp. 419-422, Aug. 1996.

[30] P. Meer, D. Mintz, A. Rosenfeld, and D. Y. Kim, "Robust regression methods for computer vision: A review," Int. J. Comput. Vision, vol. 6, pp. 59-70, 1991

[31] M. J. Mirza and K. L. Boyer, "Performance evaluation of a class of M-estimators for surface parameter estimation in noisy range data," IEEE Trans. Robotics Automat., vol. 9, pp. 75-85, Feb. 1993.

[32] S. I. Olsen, "Epipolar line estimation," in Proc. Second Eur. Conf. Comput. Vision, 1992, pp. 307-311.

[33] J. Park, B. Jiang, and U. Neumann, "Vision-based pose computation: Robust and accurate augmented reality tracking," in Proc. IEEE Int. Workshop Augmented Reality, 1999, pp. 3-12.

[34] S. Russel and P. Norvig, Artificial Intelligence. Englewood Cliffs, NJ: Prentice-Hall, 1995, p. 75.

[35] M.-C. Shie et al., "Fast, robust block motion estimation using simulated annealing," IEICE Trans. Fundamentals, vol. E83, pp. 121-127, 2000.

[36] D.-G. Sim, O.-K. Kwon, and R.-H. Park, "Object matching algorithms using robust hausdorff distance measures," IEEE Trans. Image Processing, vol. 8, pp. 425-429, Mar. 1999.

[37] P. Simard, Y. Le Cun, and J. Denker, "Efficient pattern recognition using a new transformation distance," in Neural Information Processing Systems, S. J. Hanson, J. D. Cowan, and C. L. Giles, Eds. San Francisco, CA: Morgan Kaufmann, 1993, vol. 5, pp. 50-58. 
[38] C. V. Stewart, "Robust parameter estimation in computer vision," SIAM Rev., vol. 41, pp. 513-537, 1999.

[39] S. Zhu and K.-K. Ma, "A new diamond search algorithm for fast blockmatching motion estimation," IEEE Trans. Circuit Syst. Video Technol., vol. 9, pp. 287-290, Mar. 2000.

[40] Z. Zhang, "Parameter estimation techniques: A tutorial with application to conic fitting," Image Vision Comput., vol. 15, pp. 59-76, 1997.

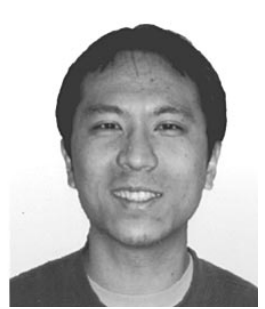

Jiun-Hung Chen received the B.S. and the M.S. degrees in computer science and information engineering from National Taiwan University, Taipei, Taiwan, R.O.C., in 1997 and 1999, respectively.

$\mathrm{He}$ is currently a research assistant in the Institute of Information Science, Academia Sinica, Taipei. His research interests include pattern recognition, image processing, and computer vision.

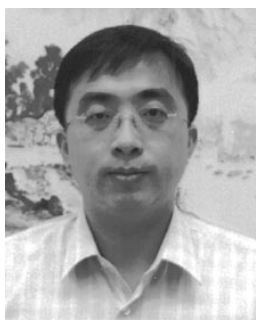

Chu-Song Chen received the B.S. degree in control engineering from National Chiao-Tung University, Hsing-Chu, Taiwan, R.O.C., in 1989 and the M.S. degree in 1991 and the Ph.D. degree in 1996, respectively, both from the Department of Computer Science and Information Engineering, National Taiwan University, Taipei.

From 1997 to 1999 , he has been a postdoctoral fellow of the Institute of Information Science, Academia Sinica, Taipei, and has been an assistant research fellow since 1999 . He has been an adjunct assistant professor with the Department of CSIE, National Taipei University of Technology since 2000. He has also been a joint appointment assistant professor with the Department of Graphic Arts and Communication, National Taiwan Normal University, Taipei, since 2001. His research interests include pattern recognition, computer vision, signal/image processing, and computer graphics.

Dr. Chen received both the outstanding paper award of the Image Processing and Pattern Recognition (IPPR) Society and the best paper award of the Image Processing and Application Association (IPAA) of Taiwan in 1997. He has received the outstanding paper award in the field of computer applications at ICS'2000, Chiayi, Taiwan.

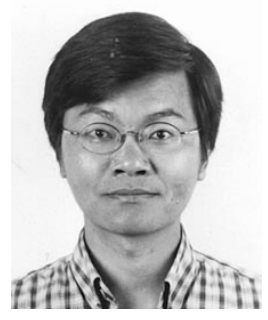

Yong-Sheng Chen received the B.S. degree in computer and information science from National Chiao Tung University, Hsinchu, Taiwan, R.O.C., in 1993 and the M.S. and the Ph.D. degrees in computer science and information engineering from National Taiwan University, Taipei, in 1995 and 2001, respectively.

He was a postdoctoral research fellow with the Institute of Information Science, Academia Sinica, Taipei, where he was engaged in computer vision research from 2001 to 2002. He is currently a postdoctoral research fellow with the Integrated Brain Research Laboratory, Department of Medical Research and Education, Taipei Veterans General Hospital. His research interests include bioelectromagnetic signal processing, medical image processing, fast template matching, pattern recognition, object detection, and visual tracking.

Dr. Chen was a recipient of the IICM Best Thesis Award and the Dragon Thesis Award in 2001 